\title{
Notch1 activity in the olfactory bulb is odour-dependent and contributes to olfactory behaviour
}

\author{
Emanuele Brai, ${ }^{1}$ Swananda Marathe, ${ }^{1}$ Lorena Zentilin, ${ }^{2}$ Mauro Giacca, ${ }^{2}$ Johannes Nimpf, ${ }^{3}$ Robert Kretz, ${ }^{1}$ \\ Alessandra Scotti ${ }^{1,4}$ and Lavinia Alberi ${ }^{1}$ \\ ${ }^{1}$ Unit of Anatomy, Department of Medicine, University of Fribourg, Route de Gockel, 1, Fribourg, Switzerland \\ 2International Centre for Genetic Engineering and Biotechnology, Trieste, Italy \\ ${ }^{3}$ Max F. Perutz Laboratories, Vienna Bio Center, Department of Medical Biochemistry, Medical University of Vienna, Vienna, \\ Austria \\ ${ }^{4}$ Unit of Anatomy, Department of Medicine, University of Bern, Bern, Switzerland
}

Keywords: mitral cells, neuronal activity, Notch1, olfactory avoidance, olfactory bulb

\begin{abstract}
Notch signalling plays an important role in synaptic plasticity, learning and memory functions in both Drosophila and rodents. In this paper, we report that this feature is not restricted to hippocampal networks but also involves the olfactory bulb (OB). Odour discrimination and olfactory learning in rodents are essential for survival. Notch1 expression is enriched in mitral cells of the mouse $\mathrm{OB}$. These principal neurons are responsive to specific input odorants and relay the signal to the olfactory cortex. Olfactory stimulation activates a subset of mitral cells, which show an increase in Notch activity. In Notch1cKOKIn mice, the loss of Notch1 in mitral cells affects the magnitude of the neuronal response to olfactory stimuli. In addition, Notch1cKOKIn mice display reduced olfactory aversion to propionic acid as compared to wildtype controls. This indicates, for the first time, that Notch1 is involved in olfactory processing and may contribute to olfactory behaviour.
\end{abstract}

\section{Introduction}

Notch signalling is a highly conserved signalling pathway which is essential for cell to cell communication from development to adulthood (Artavanis-Tsakonas et al., 1999). Notch1 is a transmembrane receptor which is activated by the interaction with specific ligands of the Jagged and Delta-Serrate family member proteins. The ligand-receptor interaction triggers sequential processing of Notch1 in the Notch intracellular domain (NICD1), which is translocated to the nucleus to form a transcriptional active complex through the binding with RBPJK (Ilagan et al., 2011). The Notch1 receptor and its cognate ligands are expressed in postmitotic neurons of the mature mouse brain (Stump et al., 2002), and there is increasing evidence that Notch signalling is involved in synaptic communication and plasticity. We have previously shown that Notch signalling occurs in response to an increase in synaptic activity, and it is required for synaptic plasticity and spatial learning in mice (Alberi et al., 2011). More recently, a study has indicated that mice lacking Notch/RBPJK signalling display an olfactory preference deficit (Sato et al., 2012), suggesting that Notch signalling may also be involved in olfactory processing. Interestingly, in Drosophila, Notch signalling is responsive to odour stimulation (Lieber et al., 2011) and it is required for olfactory learning (Zhang et al., 2013).
Olfaction is highly conserved among species and is essential for survival and reproduction (Kaupp, 2010). In rodents, a specific odour activates a set of olfactory sensory neurons in the olfactory epithelium that project to discrete glomeruli within the olfactory bulb (OB) (Mori et al., 2006). The neural signal is then conveyed to underlying mitral cells (MCs), which relay the signal to the piriform cortex, the entorhinal cortex and the amygdala (Mori \& Yoshihara, 1995). The physiological properties of the olfactory circuit have been well characterised; nevertheless, few reports have addressed signalling mechanisms regulating neuronal communication and olfactory behaviour (Cao et al., 2012; Hellwig et al., 2012; Néant-Fery et al., 2012; $\mathrm{Xu}$ et al., 2013). Interestingly, olfaction is the first sense which is affected in Alzheimer's disease (Rahayel et al., 2012) and it has been proposed that alteration in synaptic transmission may underlie the olfactory deficit. Based on the studies pointing at an alteration in Notch signalling in progressive neurodegenerative diseases (Berezovska et al., 1998; Nagarsheth et al., 2006) we hypothesised that Notch signalling may contribute to olfactory coding. In the present study we show that Notch1 activity in MCs is odour dependent and that loss of Notch1 affects odour processing and olfactory avoidance.

\section{Materials and methods}

\section{Animals}

Experiments were performed on adult male wildtype and transgenic mice on a C57BL/6 background (25-30 g; 2-3 months old). Mice 
mutant for Notch1 in excitatory neurons of the forebrain were obtained by crossing the Notch1flox/flox (Radtke et al., 2000) mice with the $\operatorname{Tg}($ CamKII- $\alpha:: c r e) 159 \mathrm{Kln}$ mouse line (Minichiello et al., 1999). Notch 1 flox/flox and $\operatorname{Tg}(\mathrm{CamKII}-\alpha:: c r e) 159 \mathrm{~K} \ln$ mice were included in the study as wildtype animals. Genotypes of all the mice were confirmed through PCR analysis of genomic DNA. Animals were housed under standard laboratory conditions in an air-conditioned room, with a 12-h controlled dark/light cycle, and were provided with food and water ad libitum. All the animal procedures were in accordance with the EU Directive 2010/63/EU on the protection of animals used for scientific purposes and were approved by the local Animal Care Committee (Canton of Fribourg, Switzerland).

\section{Viral reagents}

pAAV-CBFREwt-DIO-EYFP-WPRE-pA and pAAV-CBFREmutDIO-EYFP-WPRE-pA (as negative control) were cloned from the pAAV-DIO-eNpHR-EYFP-WPRE-pA (Addgene, Cambridge, US; no. 20949) and CBFREwt and CBFREmut constructs (Mizutani et al., 2007). AAV with serotype 9 efficiently infects post-mitotic neurons (Aschauer et al., 2013). The viral titre for in vivo injection was $10^{8-12} / \mathrm{mL}$.

\section{Viral injections}

Five $\operatorname{Tg}($ CamKII- $\alpha:: c r e) 159 K \ln$ and three N1cKOKln mice were injected in the OB with the viral construct pAAV9-CBFREwt-DIOEYFP-WPRE-pA. Two $\mathrm{Tg}$ (CamKII- $\alpha:$ :cre $) 159 \mathrm{Kln}$ mice and one N1cKOKln were injected with the mutant viral construct pAAV9CBFREmut-DIO-EYFP-WPRE-pA. The mice were weighed and thereafter deeply anesthetised with an intraperitoneal injection of a mixture of ketamine (stock $100 \mathrm{mg} / \mathrm{mL}$ Ketanarkon; Streuli Pharma AG, Uznach, Switzerland) and xylazine (stock $20 \mathrm{mg} / \mathrm{mL}$ Xylazine; Streuli Pharma AG) at a final concentration of 100 and $10 \mathrm{mg} / \mathrm{kg}$ in sterile saline solution ( $\mathrm{NaCl}$ 0.9\%; Braun Medical SA, Sempach, Switzerland). The withdrawal reflex was checked every $30 \mathrm{~min}$ to monitor anesthesia. The viruses were administered by using a Hamilton syringe $(5 \mu \mathrm{L}$; Hamilton Company, Bonaduz, Switzerland) with a 34-gauge needle attached. The coordinates for injections were - anteroposterior (AP), $+4.7 \mathrm{~mm}$ from bregma; mediolateral (ML), $+1.0 \mathrm{~mm}$ from the midline; and dorsoventral (DV), $-0.2 \mathrm{~mm}$, according to the Allen brain atlas coordinates (http:// mouse.brain-map.org/). The needle was maintained in position for $1 \mathrm{~min}$ after the injection to avoid any backflow of virus. One week later, mice were exposed to a novel odour as previously described (Bepari et al., 2012) - mice were left for $2 \mathrm{~h}$ in a clean empty cage with overhead airflow, without food and water before the odorant exposure. Pure odorant was collected in a $1.5-\mathrm{mL}$ tube and fixed on the internal wall of the cage. The molecules used were amyl acetate (60 $\mu \mathrm{L}, 99 \%$; Sigma-Aldrich, USA) and tap water $(60 \mu \mathrm{L})$ as control. Mice were exposed to the odorant for $25 \mathrm{~min}$ and killed $3 \mathrm{~h}$ later for histological analysis (Fig. 4B).

\section{Surgery and extracellular in vivo recordings}

Extracellular recordings were carried out on three wildtypes [two Tg (CamKII- $\alpha::$ cre) 159 Kln and one Notch1flox/flox] and three Notch1cKOKln mice. Mice were deeply anesthetised with an intraperitoneal injection of a mixture of ketamine and xylazine as above. The withdrawal reflex was checked every $30 \mathrm{~min}$ to monitor anesthesia. The body temperature was maintained at $37.5{ }^{\circ} \mathrm{C}$ using a heating pad connected to a temperature sensor placed intrarectally (Lis-Medical, Frankfurt, Germany). The mouse was mounted in a stereotaxic frame for surgery (Kopf Instruments, Tujunga, CA, USA). The surface of the skull above the $\mathrm{OB}$ was cleaned and an incision was made to open a small window on top of the OB. Single glass-coated, platinum- and gold-plated electrodes with low impedance (1-2 M $\Omega$ ) were used for extracellular recordings (Albéri et al., 2013). The electrodes were advanced with a $45^{\circ}$ angle from the right side into the dorsal $\mathrm{OB}$ to reach the mitral cell layer (MCL) using a micromanipulator (Kopf Instruments) at the coordinates AP, +4.05 to $+4.95 \mathrm{~mm}$ from bregma, ML, $1.2-1.5 \mathrm{~mm}$ and DV, 200$600 \mu \mathrm{m}$. After the first penetration the surface of the OB was protected using a $3 \%$ pure agarose gel (Carl Roth GMBH + Co. KG, Karlsruhe, Germany). An average of six electrode penetrations were performed in the mid-anterior OB per animal. The signal from the electrode was amplified and filtered (400-2000 Hz), visualised on an oscilloscope, conveyed to an amplifier (Power 1401; Cambridge Electronic Design Limited, Cambridge, UK). The signal was digitised (10 kHz sampling rate) using Spike 2 (Cambridge Electronic Design Limited). The signals were recorded for $\sim 1 \min 30 \mathrm{~s}$ of spontaneous activity followed by $1 \mathrm{~s}$ of odour stimulation and $30 \mathrm{~s}$ of evoked activity. A volume of $50 \mu \mathrm{L}$ of amyl acetate (99\%; Sigma-Aldrich) and benzaldehyde (98\%; Sigma-Aldrich) was pipetted into a $1.5-\mathrm{mL}$ tube and presented at $2 \mathrm{~cm}$ distance from the snout. The estimated concentrations of amyl acetate and benzaldehyde in the air were 4.4 and $1.5 \mu \mathrm{m}$ respectively. The 1-s odour exposure was recorded as a triggered signal. Following the recording session electrolytic lesions were induced at specific depths using three pulses of $5 \mathrm{~mA}$ for $7 \mathrm{~s}$ at intervals of $5 \mathrm{~s}$.

\section{Antibodies}

The primary antibodies used in the immunohistochemistry were goat anti-Notch1 (sc6014; Santa Cruz Biotechnology, Inc., Dallas, TX, USA), rabbit anti-Jagged1 (Ab7771; Abcam plc, Cambridge, UK), rabbit anti-Tbr2 (Ab23345; Abcam), mouse anti-Reelin (MAB5364; Millipore, Billerica, MA, USA), mouse anti-GFAP (NBP105197; Novus Biologicals Ltd., Cambridge, UK) and rabbit anti-c-fos (PC38; Calbiochem, San Diego, CA, USA). The primary antibodies used for Western blot were rabbit anti-Notch1 (07-220; Millipore), mouse anti-Jagged1 (H00000182-M01A; Novus Biologicals Ltd.), rabbit anti-c-fos (PC38; Calbiochem) and mouse anti- $\beta$-tubulin (T5168; Sigma-Aldrich).

The secondary antibodies used for the fluorescence immunohistochemistry were Cy2 donkey anti-mouse (1 : 600; cat. no. 715-545150), Cy2 donkey anti-rabbit (1:600; cat. no. 711-545-152), Су3 donkey anti-goat (1 : 600; cat. no. 705-165-147), Cy3 donkey antirabbit (1: 500; cat. no. 711-165-152) and Cy5 donkey anti-mouse (1 : 500; cat. no. 715-605-150). All fluorescent conjugated antibodies were purchased from Jackson Immunoresearch Europe Ltd., Newmarket, Suffolk, UK. Chromogenic immunohistochemistry was performed using the biotinylated donkey anti-goat $(1: 600$; cat. no. 705-066-147, Jackson Immunoresearch Europe Ltd.), streptavidin conjugated to horseradish (HRP; cat. no. BA-9500; Vector Laboratories, Liestal, Switzerland) and 3-3'-diamminobenzidine (Thermo Fisher Scientific Inc., Waltham, MA, USA). The secondary antibodies used for the immunoblots were infrared-dye-conjugated (IR-Dye) - donkey anti-mouse IgG IR800 (cat. no. 926-32212; LI-COR Biosciences $\mathrm{GmbH}$, Bad Homburg, Germany), goat anti-mouse IgG IR680 (cat. no. 926-68070; LI-COR Biosciences GmbH) and donkey anti-rabbit IgG IR800 (cat. no. 926-32213; LI-COR Biosciences $\mathrm{GmbH})$. All IR-antibodies were diluted 1:10 000 . 


\section{Histology and immunohistochemistry}

All mice were anesthetised using a dose of $10 \mu \mathrm{L} / \mathrm{g}$ of ketamine/xylazine and rinsed transcardially with $50 \mathrm{~mL}$ of $0.9 \% \mathrm{NaCl}$ to wash out the blood and then fixed with $4 \%$ paraformaldehyde (PFA) in $0.1 \mathrm{M}$ phosphate-buffered saline (PBS). The brains were left immersed in $4 \%$ PFA overnight on a rotating shaker at room temperature. One day later, the brains were cryoprotected in $30 \%$ sucrose in $0.1 \mathrm{~m} \mathrm{PBS}$, at room temperature. Thereafter, the brains were mounted in OCT (Takara Bio Inc., Otsu, Shiga, Japan) and blocks were sectioned on a cryostat (Leica Microsystems GmbH, Wetzlar, Germany) to obtain floating coronal, and in some instances sagittal, sections of 30-50 $\mu \mathrm{m}$ thickness. Horizontal sections for chromogen immunohistochemistry were cut on a vibratome (Microm International $\mathrm{GmbH}$, Walldorf, Germany) using a skewed angle (Markopoulos et al., 2008). Nissl staining was performed on $\mathrm{OB}$ sections from mice used for the extracellular recordings to visualise the electrolytic lesions. Immunohistochemical stainings were performed on sections from the $\mathrm{OB}$ of viral-injected mice and on sagittal sections from brain of wildtype, N1cKOKln and $\operatorname{Tg}($ CamKII- $\alpha:: c r e) 159 K \ln$ mice as previously described (Alberi et al., 2011). Images were obtained using a Leica TCS SP5 Confocal (Leica Microsystems GmbH) with $20 \times, 40 \times$ and $63 \times$ objectives and a Leica DM6000B (Leica Microsystems $\mathrm{GmbH})$ automated upright microscope with a $20 \times$ objective. Each immunohistochemistry experiment was performed from three to five times per immunostaining.

Notch1 expression in MCs, granule cells (GCs) and periglomerular cells (PGCs) of wildtype animals was quantified on stacked fluorescent confocal images from five different stainings. A total of 50 cells per population was analysed. Around the cell bodies of the cells a region of interest of constant area was drawn. The mean grey value of the Notch1 signal was considered in the analysis. C-fos-immunopositive neurons were quantified on stacked confocal images from OB sections from Notch1cKOKln and wildtype mice. Three consecutive sections were imaged per animal ( $n=4$ per genotype).

\section{Western blot}

The OB of four wildtype (non-transgenic and Notch1flox/flox) and five N1cKOKln mice were homogenised in Nonidet P-40 lysis buffer supplemented with a protease inhibitor cocktail (1 : 100; Carl Roth GMBH + Co. KG). Lysates were processed for Western blot as previously described (Towbin et al., 1979). For the detection of the proteins, low-fluorescence PVDF membranes (BioRad Laboratories Inc., Hercules, CA, USA) were incubated with the primary antibodies overnight at $4{ }^{\circ} \mathrm{C}$ with gentle agitation. On the second day, the membranes were rinsed with Tris-buffered saline solution supplemented with $1 \%$ Tween (TBST) and then incubated for $1 \mathrm{~h}$ with IR-Dye secondary antibodies, rinsed again and dried. Signals were detected in the 700- and 800-nm channels using the LI-COR Odyssey Infrared Imager (LI-COR Biosciences GmbH). The Western blot assay on the $\mathrm{OB}$ of Notch1cKOKln and wildtype mice was performed four times. The optical density of each band was determined using Image $\mathbf{J}$ software and normalised to the $\beta$-tubulin control. Student's $t$-test was performed to compare normalised optical density values between genotypes.

\section{Olfactory behaviour}

Olfactory behaviour was tested on up to nine wildtype (Notch1flox/ flox) and nine N1cKOKln adult mice (2-3 months old). The experiments were carried out using two cohorts of mice - the first cohort consisted of four wildtype and four N1cKOKln mice and the second cohort up to five wildtype and five N1cKOKln mice. The protocols followed were according to the guidelines of the Behavioral Core of the Johns Hopkins University School of Medicine. The olfaction experiment was performed in three consecutive weeks in which the mice were tested for a different task. Prior to the start of every experiment, the mice were habituated for $5 \mathrm{~min}$ in a clean cage containing only extra bedding ( $\sim 3 \mathrm{~cm}$ deep) and then were employed in the olfaction task. In the first task measuring latency to find a novel odour, a small cube of Swiss cheese was hidden under the bedding on the side opposite where the mouse was introduced (Fig. 8E). The time spent to find the cheese in a time limit of $5 \mathrm{~min}$ was counted using a digital stopwatch. The stopwatch was stopped when the mice had their nose and whiskers buried in the bedding above the cheese.

In the second task measuring time spent sniffing a novel odour, $60 \mu \mathrm{L}$ of vanilla and $60 \mu \mathrm{L}$ of tap water were pipetted onto opposite walls of the cage and within 2 min of exposure to the novel odour the time spent sniffing the vanilla aroma was recorded with the digital stopwatch (Fig. 8F).

The third task, measuring avoidance of two unpleasant odours, was performed following the same procedure as the second one but, instead of vanilla, $60 \mu \mathrm{L}$ of propionic acid (99.5\%; Sigma-Aldrich) and 2-methylbutyric acid (98\%; Sigma-Aldrich) were presented (Fig. $8 \mathrm{~F})$. All behavioural experiments were video-recorded. The behavioural data were analysed post hoc using the GRAPHPAD PRISM 5 software. Student's $t$-test was used to compare the behavioural performance between genotypes.

\section{Analysis of electrophysiological data}

The recorded waveforms were analysed post hoc from electrode penetration sites in the MCL only. Spike units corresponding to one cell were sorted from each recording using Spike 2. Peristimulus time histograms for amyl acetate and benzaldehyde were calculated for all sorted spike traces using the Neuroexplorer software (Nex Technologies, Madison, AL, USA). The mean spontaneous firing rate for each cell was obtained from the autorenewal density plot using the Neuroexplorer software. Cross-correlation analysis was performed between each cell pair in the multicellular recordings using the Neuroexplorer software. A cross-correlogram characterised by a symmetric hump near lag 0 indicated that the cells fire synchronously. Cross-correlograms with no hump or an asymmetric hump near lag 0 were characterised as non-synchronous. The mean spontaneous firing rate and the peristimulus response were compared between genotypes using Student's $t$-test. The distributions between cell pairs firing synchronously and asynchronously, in the presence of amyl acetate and benzaldheyde, in the wildtypes and in Notch1cKOKln, were analysed using two-way ANOVA.

\section{Statistical analysis}

Homogeneity of variance within groups and genotypes was verified by Bartlett's test. Differential expression of Notch1 in MCs, PGCs and GCs was analysed using one-way ANOvA (main factor, Notch1 expression; three groups, MCs, PGCs and GCs). Comparisons, between wildtype and Notch1cKOKln mice, in protein expression, c-fos-positive cells, behavioural performances, firing rate and cumulative frequencies of MCs were carried out using non-directional Student $t$-test. The peristimulus responses was analysed by one-way ANOVA with two factors, odour-evoked responses and synchrony by two-way ANOva. Quantifications reported on the text or graphs represent averages with standard errors of the mean (SEM). On graph 
charts a single asterisk $(*)$ indicates a $P$ value lower than 0.05 , double asterisks $\left({ }^{*}\right)$ indicate a $P$ value lower than 0.01 and triple asterisks $(* * *)$ indicate a $P$ value lower than 0.005 . Statistical analysis were performed using Excel Data Analysis Package and GRAPH PAD PRISM 5.

\section{Results}

\section{Notch1 and Jagged1 expression in the $O B$}

To visualise the presence of Notch1 in neurons of the OB, we performed chromogen immunolabelling using a validated Notch1 antibody. We observed that Notch1 was expressed in discrete layers of the OB (Fig. 1A) with strongest labelling in the MCL (Fig. 1A'; mean grey value for Notch1, $48 \pm 7.7)$. Notch1 was also expressed at lower levels in the periglomerular layer (PGL; mean grey value for Notch1, $25 \pm 3.5$ ) and sparse cells in the external plexiform layer (EPL), with lowest expression in the granule cell layer (GCL; mean grey value for Notch1, $15 \pm 2.9$; differential expression of Notch1 in MCs, PGCs and GCs, $F_{2,12}=18.59$, $P=0.0002$; Fig. $1 \mathrm{~A}^{\prime}$ ). To verify that Notch 1 is expressed in glutamatergic MCs, we performed a double immunolabelling with Notch1 and Tbr2 (Fig. 1B). We observed that the Notch1-positive cells were also Tbr2-positive although, as expected, expression of Tbr2 was variable (Mizuguchi et al., 2012). To further confirm the identity of the Notch-positive MCs we performed a co-labelling with Notch1 and Reelin. Reelin is constitutively expressed in MCs and acts as detachment signal for migrating neuroblasts (Hack et al., 2002). We observed that Notch1-positive cells were also strongly labelled for Reelin (Fig. 1C). These data indicate that Notch1 may be functionally relevant to the principal neurons of the OB.

As MCs receive direct input from olfactory receptor neurons, we further investigated the localisation of the cognate ligand Jagged 1 in the olfactory bulb. Interestingly, we observed that Jagged1 was strongly expressed in the olfactory nerve layer (Fig. 2A, white

\section{Notch1}

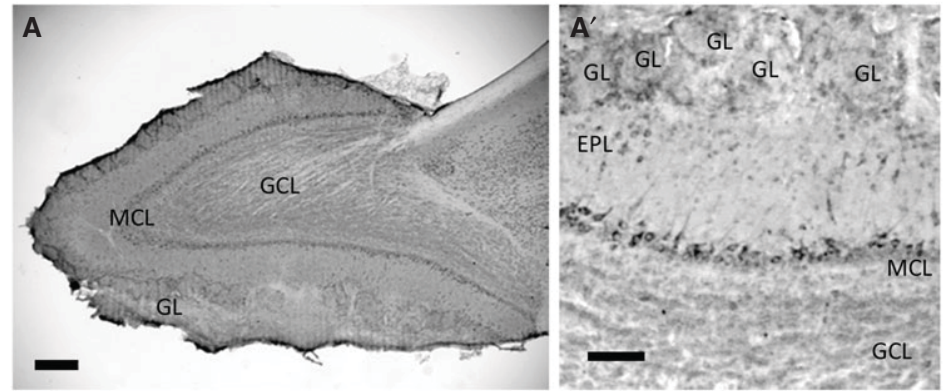

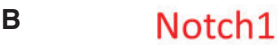

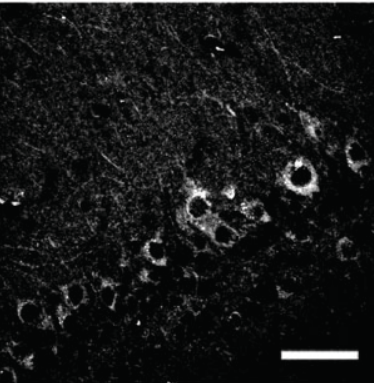

C

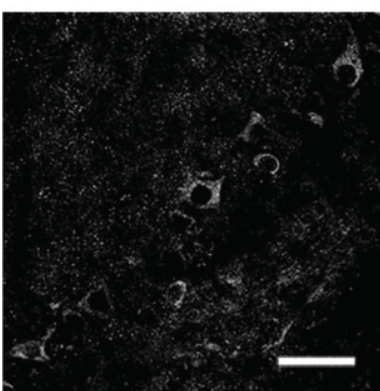

Tbr2

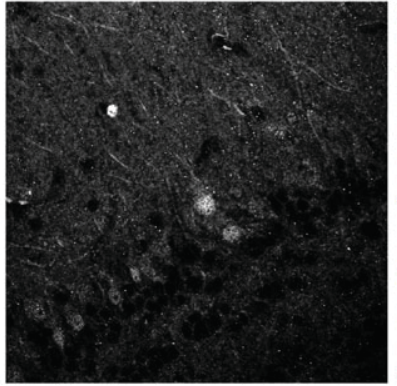

Reelin

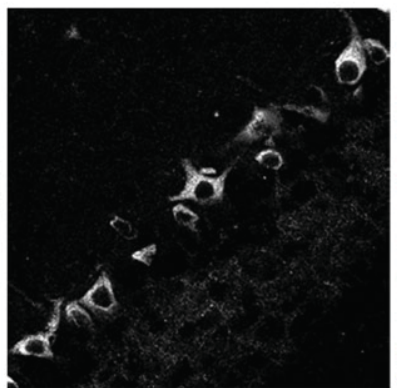

Overlay

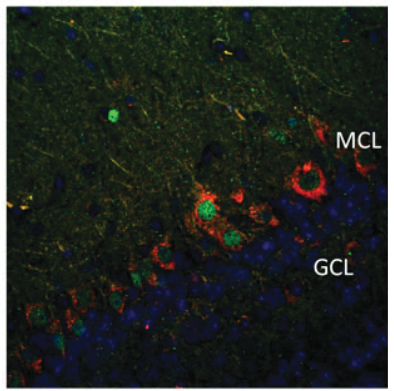

Overlay

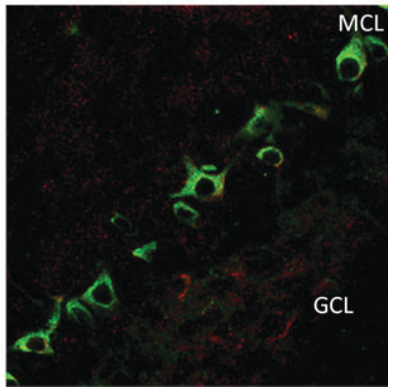

FIG. 1. Notch1 localises in the neurons of the adult olfactory bulb. (A) Chromogen immunohistochemistry shows that Notch1 (black) is expressed in different layers of the OB. $\left(\mathrm{A}^{\prime}\right)$ Close up of the OB shows that Notch1 (black) is enriched in the soma of cells in the MCL. Lower expression of Notch1 is visible in the PGCs, GCs and in sparse cells of the EPL. (B) Notch1-positive cells (red) also express the MC marker Tbr2 (green). (C) Notch1 (red) is observed in MCs, hall-marked by Reelin (green) expression. EPL, external plexiform layer; GCL, granule cell layer; GL, glomerulus; MCL, Mitral cell layer; PGC, periglomerular cell. Scale bars, $200 \mu \mathrm{m}(\mathrm{A}), 50 \mu \mathrm{m}$ ( $\mathrm{A}^{\prime}$ and B), $25 \mu \mathrm{m}(\mathrm{C})$. 
A

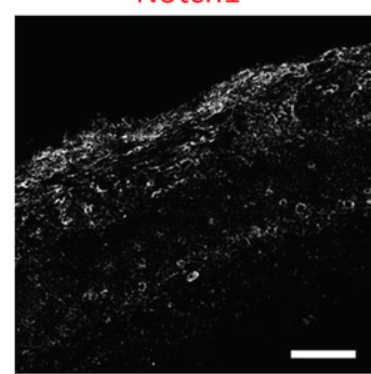

$\mathbf{A}^{\prime}$

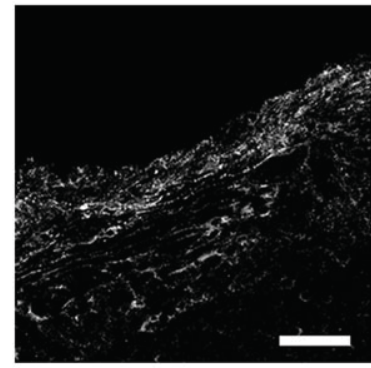

Jagged1
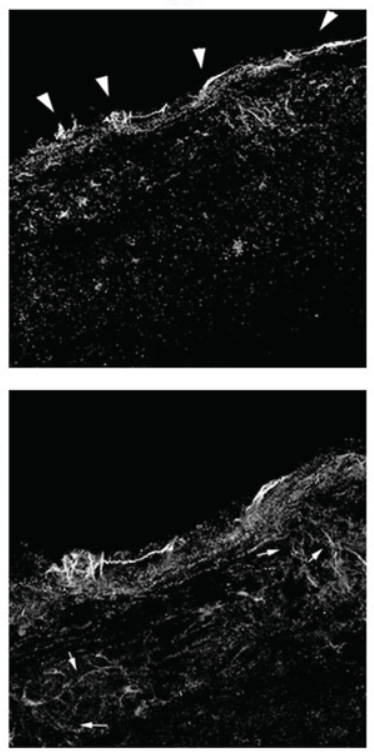

Overlay + DAPI
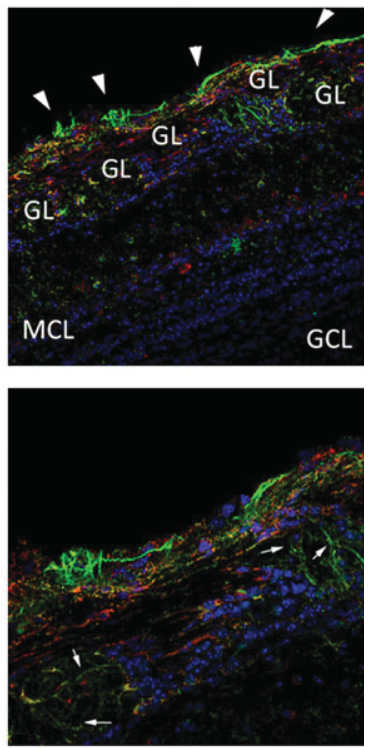

B

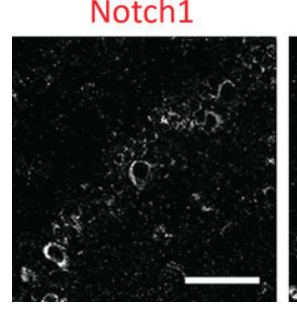

Jagged1

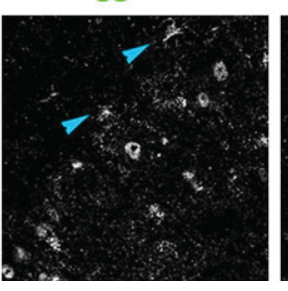

GFAP

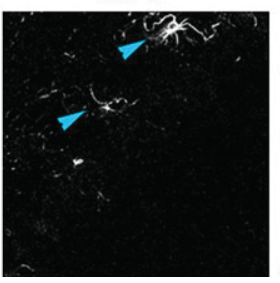

Overlay

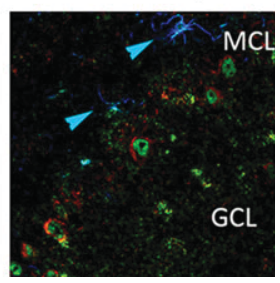

FIG. 2. Jagged1 is enriched in the olfactory nerve layer. (A) Jagged1 (green) expression is visible on the outer surface of the glomeruli (white arrowheads), whereas Notch1 (red) is present in the PGCs. ( $\left.\mathrm{A}^{\prime}\right)$ Close-up view of the glomerular boundary indicates that Jagged1 (green) is localised in filamentous structures extending from the olfactory nerve layer and invading the GL (white arrows). Notch1 (red) is localised on soma and processes of the PGCs. (B) Jagged1 (green) co-localises with Notch1 (red) in MCs. Jagged1 is also expressed in resident glia (GFAP, blue) of the OB (blue arrowheads). EPL, external plexiform layer; GCL, granule cell layer; GL, glomerulus; MCL, mitral cell layer; PGC, periglomerular cell. Scale bars, $50 \mu \mathrm{m}$ (A and $\left.\mathrm{A}^{\prime}\right), 25 \mu \mathrm{m}(\mathrm{B})$.

arrowheads). A higher magnification of the glomerular layer shows that Jagged1 is present on single processes invading few glomeruli (Fig. 2A', white arrows). Further, we observed that Jagged1 is also expressed in cells of the MCL and GCL with punctate staining in the GCL (Fig. 2B). Thus, MCs express both the Notch1 receptor and the Jagged1 ligand. Jagged1 is also observed in the soma of sparse resident, GFAP-labelled, glia (Fig. 2B, blue arrowheads). The expression pattern of Jagged 1 has been confirmed using different antibodies for Jagged1 (data not shown). Each antibody was further validated in our newly generated Jagged1cKO mouse lines.

This data indicates that Jagged1 and Notch1 appear to have functionally relevant distribution patterns as Jagged1 labels the olfactory nerve layer and is observed in putative nerve fibres within discrete glomeruli. In addition, Notch1 co-localises with Jagged1 in principal cells of the OB.

\section{Novel odour exposure induces Notch activity in MCs}

Based on the evidence that Notch1 is expressed in MCs and that signalling can be induced by synaptic activity (Alberi et al., 2011), we addressed whether exposure to the novel odour, amyl acetate, can induce Notch activation in dorsal MCs. Odorant maps for a variety of odours have been extensively documented (Mori et al., 2006). We chose amyl acetate because it induces robust $\mathrm{OB}$ activation of dorsal and ventral glomeruli (Xu et al., 2003) and underlying networks (Willhite et al., 2006). We took advantage of a multimeric Notch transcriptional reporter (Mizutani et al., 2007) and cloned it into an AAV-double floxed (DIO) vector containing the yellow-fluorescent protein (YFP) reporter (Gradinaru et al., 2008) (Fig. 4A). Viral particles were packaged and injected into the OB of $\operatorname{Tg}(\mathrm{CamKII}-\alpha:$ :cre $)$ 159Kln mice (Minichiello et al., 1999) (Fig. 4B). We confirmed that CamKII- $\alpha$ expression is prevalent in MCs (Liu, 2000) (Fig. 3). This allows inversion of the reporter in the orientation of transcription in CamKII- $\alpha:$ :cre-expressing neurons; nevertheless, YFP expression is only visible in Notch-activated cells (Fig. 4A). The specificity of the Notch-dependent YFP signal in the MCs was confirmed using three methods: (i) exposure to the neutral and familiar odour, water (Fig. 4C and E); (ii) a mutant responsive element, which is not responsive to Notch/RBPJK signalling (Fig. 4F) and (iii) a transgenic Notch1 loss-of-function model, the Notch1cKOKln, in which Notch1 is depleted in all mature neurons of the forebrain (Fig. 4G).

One week following viral injection, the mice were exposed to amyl acetate or water and killed $3 \mathrm{~h}$ later (Fig. 4B). We observed that $\operatorname{Tg}(\mathrm{CamKII}-\alpha:$ :cre $) 159 \mathrm{Kln}$ mice injected with the viral particles pAAV-CBFREwt-DIO-EYFP-WPRE-pA displayed YFP expression in MCs of the dorsal OB, and dorsal as well as ventral glomerular clusters, only when exposed to amyl acetate, not to water (Fig. 4CE). On the other hand, $\operatorname{Tg}(\mathrm{CamKII}-\alpha:: \mathrm{cre}) 159 \mathrm{Kln}$ mice, injected 

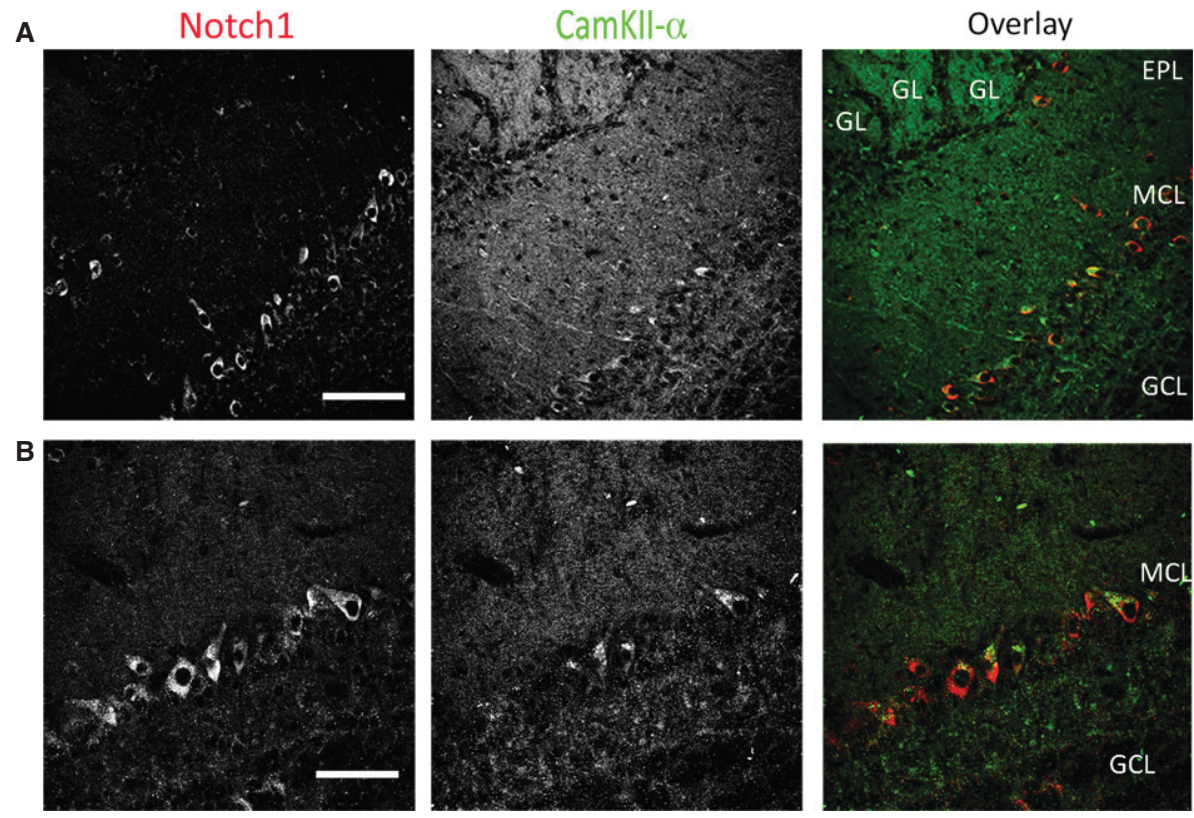

FIG. 3. CamKII- $\alpha$ expression is highest in MCs and colocalises with Notch1. (A) CamKII- $\alpha$ expression is highest in the MCL as compared to PGCs and GCs. CamKII- $\alpha$-positive processes extend into the EPL. (A and B) Notch1-positive mitral cells are also CamKII- $\alpha$ positive. EPL, External plexiform layer; GCL, granule cell layer; GL, glomerulus and MCL, Mitral cell layer. Scale bars, $50 \mu \mathrm{m}$ (A), $25 \mu \mathrm{m}$ (B).

with a mutant version of the virus, pAAV-CBFREmut-DIO-EYFPWPRE-pA, did not display any YFP expression in the MCL (Fig. 4F). In addition, in the Notch1cKOKln's OB, viral injection of the Notch-responsive virus did not yield any YFP expression after amyl acetate exposure (Fig. 4G). This indicates that Notch1 is required for this signalling activity. The specificity of the YFP reporter was confirmed by the presence of Notch1 in YFP-positive MCs (Fig. 4H).

To confirm the discrete activation of the olfactory neuronal ensembles upon odorant exposure, we adopted c-fos as a general marker for activity. Interestingly, we noticed that below the group of MCs, where YFP expression is intense as a result of amyl acetate exposure, numerous putative GC interneurons show c-fos immunoreactivity (Fig. 4I)

These results indicate that Notch signalling is activated in discrete mitral neurons and glomeruli following odorant exposure, and underline the exclusive requirement of Notch1 in MCs to trigger Notch/RBPJK transcriptional activity. This suggests that Notch signalling correlates with the degree of neural activity in the OB.

\section{Loss of Notch1 in MCs affects olfactory bulb neural activity}

Based on the evidence that Notch activity is induced in assemblies of MCs following odorant stimulation and that Notch1 appears to be essential to maintain signalling, we investigated the phenotype of the Notch1cKOKln mouse line. The Nissl stainings did not indicate any morphological abnormalities in the OB of Notch1cKOkln mice as compared to wildtypes (Fig. 6A). However, Notch1 expression was homogenously and consistently reduced, but not completely abolished, in the MCL of the Notch1cKOKln mice, indicating the specificity of the deletion (mean Grey values: $22.9 \pm 2.5$ in the knockouts vs. $48.3 \pm 5.5$ in the wildtypes; $t_{8}=4.28, P=0.002$; $n=5$ animals per genotype; Fig. 5A). Western blot analysis on whole OBs of the Notch1cKOKlns indicated that Notch1 is ablated by $30 \%$ as compared to wildtype littermate controls (Fig. 5B; $1.2 \pm 0.1$ and $1.8 \pm 0.2, t_{6}=3.242, P=0.018 ; n=4$ animals per genotype), suggesting that the deletion is specific to a subset of cells in the OB. Based on the fact that Notch1 and Jagged1 are both expressed in this region, we addressed whether Jagged1 levels were altered in the Notch1cKOKlns. The levels of Jagged1 appeared unchanged between Notch1cKOKln and wildtype, indicating that the specific reduction in Notch1 does not affect ligand expression (Fig. 5B).

As Notch1 appears to be important to synaptic plasticity, we investigated the effect of Notch1 loss on c-fos expression. Earlyimmediate genes, such as c-fos and Zif268, have been previously used to map the activity of neurons in the OB in response to odorants (Kobayakawa et al., 2007; Bepari et al., 2012). We observed that $\mathrm{c}$-fos expression was increased about two-fold in the $\mathrm{OB}$ of Notch1cKOKln as compared to wildtype mice $(2.5 \pm 0.3$ and $1.4 \pm 0.1, t_{4}=3.528, P=0.024 ; n=3$ Notch 1 cKOKlns and $n=3$ wildtypes; Fig. 5B). Immunohistochemical analysis of c-fos expression confirms that, in the absence of any stimuli, the number of c-fos-positive cells was significantly higher in the MCL and GCL of Notch1cKOKlns than in wildtypes $(37 \pm 6.2$ and $7.8 \pm 3.5$, $t_{6}=4.141, P=0.006 ; n=4$ per genotype; Fig. 5C). C-fos expression was not localised to faintly positive Notch1 mitral neurons but was evident in interneurons within the MCL and the GCL (Fig. 5C and D). This is in accordance with the increase in c-fos expression in these layers following amyl acetate stimulation (Fig. 4I).

These results suggest that, as a consequence of Notch1 loss in $\mathrm{MCs}$, the activity of neuronal assemblies in the OB may be altered.

\section{Notch1 regulates $M C$ activity and synchrony}

Based on the evidence that ablation of Notch1 induces ectopic c-fos expression, as a readout of increased neuronal activity, in a subset of granule cells within the GCL and MCL, we investigated the firing 


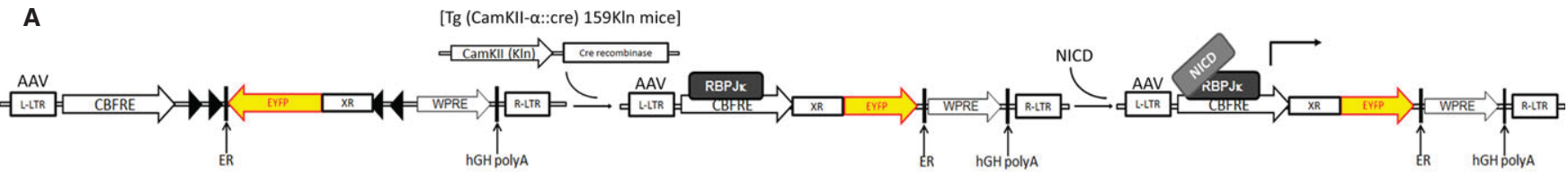
pAAV9-CBFRE-doublefloxed-EYFP-WPRE-pA

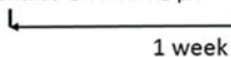
25' Amyl acetate la
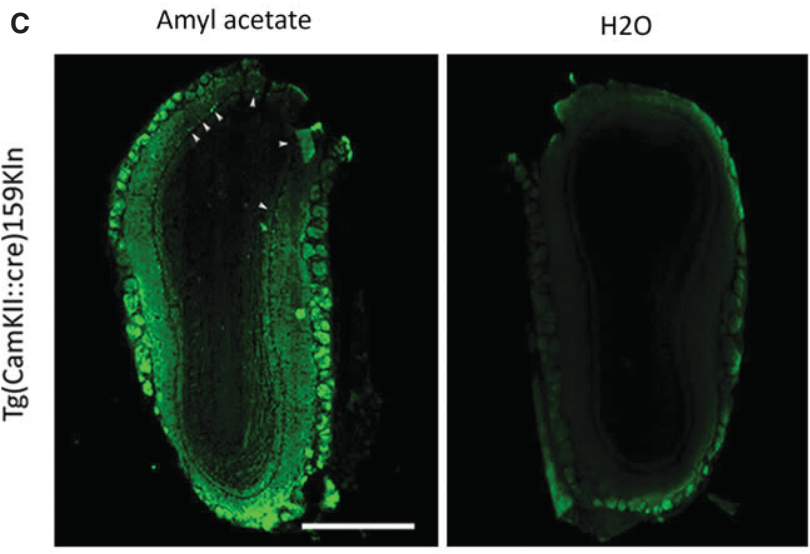

H AAV-CBFwtRE::YFP
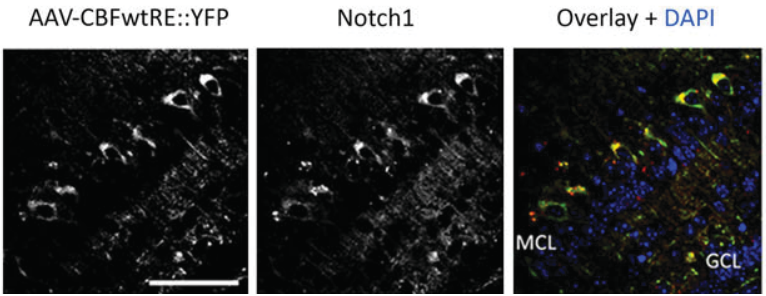

Sacrifice

h

$\longrightarrow$

FIG. 4. Odorant stimulation induces Notch signalling in MCs. (A) Experimental strategy for conditional targeting of CamKII::cre-expressing cells using an AAV with a Notch-responsive element (CBFRE), which drives EYFP as a result of Notch activation (NICD). (B) Scheme of experimental paradigm for viral injection of a cre- and Notch-responsive (AAV-CBFRE-flox-YFP) element in Tg(CamKII- $\alpha:$ :cre) $159 \mathrm{Kln}$ mice] and exposure to the odorant amyl acetate (Amyl ac.). (C) Notch-responsive YFP-positive neurons are visible in the dorsal OB following amyl acetate stimulation (white arrowheads) but not after water exposure. (D) YFP expression (green) is clearly visible in MCs of the OB infected with the Notch-responsive virus AAV-CBFwtRE::YFP after amyl acetate exposure. (E and F) YFP expression is absent from the OB of animals either exposed to water or infected with a mutated form of the virus AAV-CBFmutRE::YFP. (G) Three hours after amyl acetate exposure, YFP (green) is not visible in the bulb of the Notch1cKOKln [Tg(CamKII-a::cre) $159 \mathrm{Kln}$ mice] mice injected with the Notch responsive virus AAV-CBFwtRE::YFP. (H) The Notch-responsive YFP (green) is visible in MCs expressing the Notch1 receptor (red). (I) After amyl acetate exposure, YFP (green), as readout of Notch activation, is expressed in MCs and their processes crossing the GCL, where several cells display sustained c-fos expression. GCL, granule cell layer; GL, glomerulus; MCL, mitral cell layer; CBFRE, multimeric 4XCBF reporter; NICD, Notch intracellular domain; $\mathrm{XR}$, membrane receptor; ER, endoplasmic reticular sequence; hGH polyA, human growth hormone poly A sequence. Scale bars, 1 mm (C), $150 \mu \mathrm{m}(\mathrm{D}$; also applies to $\mathrm{E}-\mathrm{G}), 25 \mu \mathrm{m}$ ( $\mathrm{H}$ and $\mathrm{I})$.

properties of MCs in the Notch1cKOKlns as compared to wildtype controls $\operatorname{Tg}($ CamKII- $\alpha::$ cre $) 159 K \ln$ and Notch1flox/flox. Mapping experiments have indicated that amyl acetate activates the dorsal medial glomeruli and underlying MCs (Xu et al., 2003), whereas benzaldehyde induces activation of the lateral glomeruli (Matsumoto et al., 2010). We therefore performed extracellular recording in the dorsal MCL of the mid-anterior $\mathrm{OB}$ (AP, +4.05 to $+4.95 \mathrm{~mm}$ from bregma; ML, 1.2-1.5 mm; and DV, 200-600 $\mu \mathrm{m}$ ) and measured the response of MCs to amyl acetate as opposed to benzaldehyde (Fig. 6A, C and D). We performed five to nine electrode penetrations from the dorsal $\mathrm{OB}$ for each of the three Nocth $1 \mathrm{cKOK} \ln$ and three wildtype mice. At each electrode penetration, we recorded the evoked activity in response to a 1-s exposure of amyl acetate and benzaldehyde (Fig. 6B). The interval between presentations of the two odours was 5 minutes to allow for normalisation of the spontaneous activity. Post hoc spike train analysis revealed that from each electrode penetration three to six spike units could be distinguished (Fig. 6B). No difference in spike amplitude was detected between genotypes (data not shown). Nevertheless, the spike counts and the firing frequency within the first $200 \mathrm{~ms}$ of amyl acetate exposure, as indicated by the peristimulus dot plot and the peristimulus time histogram, appeared negligible in the Notch1cKOKln whereas in the wildtype, within $100 \mathrm{~ms}$ of the stimulus there was a two-fold increase in response (Fig. 6C and $\mathrm{C}^{\prime}$ ). The trendline in the peristimulus dot plot indicates the significant difference in the firing response to amyl acetate between Notch1cKOKlns and wildtypes $\left(F_{1,1}=27.59, P=1.1 \times 10^{-6}\right.$; Fig. $\left.6 \mathrm{C}\right)$. On the other hand, benzaldehyde elicited no response in either wildtype or $\mathrm{KO}$ mice (Fig. 6D and $\mathrm{D}^{\prime}$ ). Based on this result, we asked whether, in Notch1cKOKlns, the basal activity of MCs was also 


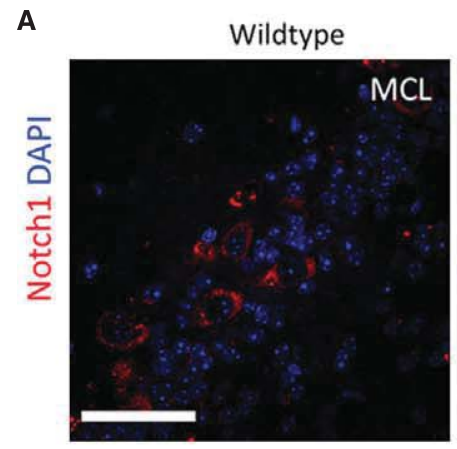

B

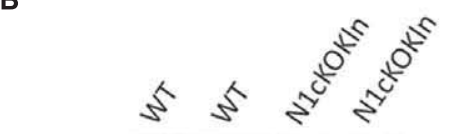

Notch1 $5 \mathrm{E}: \mathrm{ta}$ Jag1 E 4 \& 1 \& 3

c-fos

$\beta$-tubulin

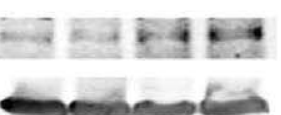

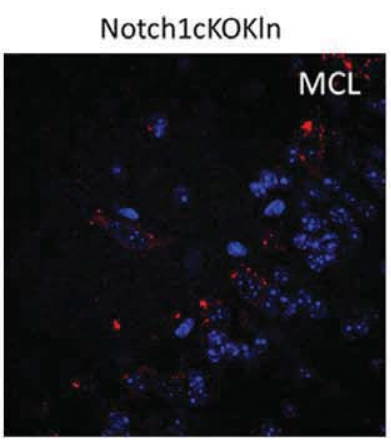
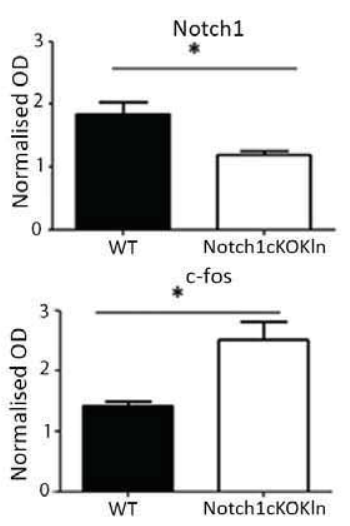

C

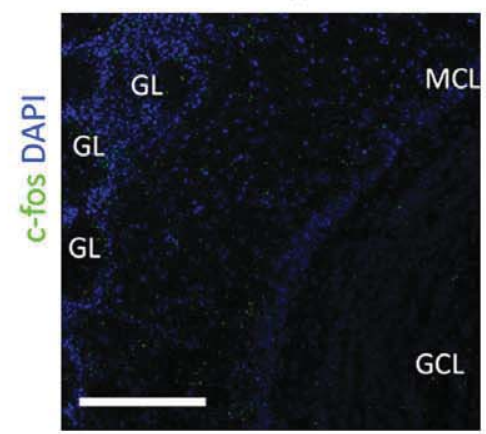

D

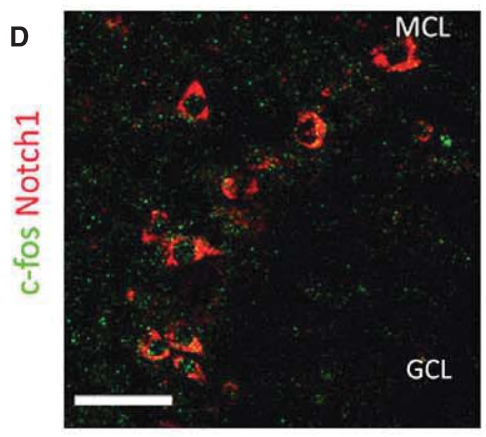

Notch1cKOKIn
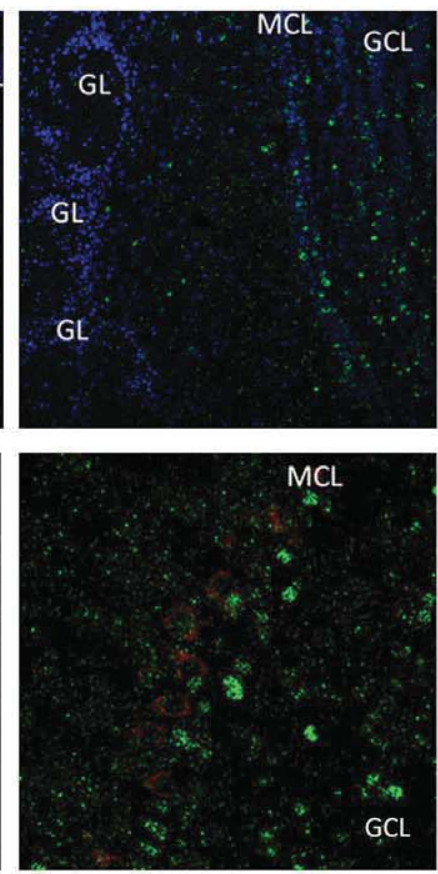

FIG. 5. The OB of Notch1cKOKln mice displays altered c-fos activity. (A) Immunohistochemistry for Notch1 (red) shows that Notch1 expression is dramatically reduced in the MCs in the Notch1cKOKln as compared to wildtype. (B) Representative Western blot analysis of OBs from two wildtype and two N1cKOKln mice indicates expression of Notch1, Jagged1 and c-fos. Charts represent the normalised expression of Notch1 and c-fos in four wildtypes and five Notch1cKOKln mice. (C) Immunohistochemistry for c-fos (green) shows that, in the knockouts, numerous c-fos-positive cells are present in the GCL and MCL as compared to wildtype in baseline conditions. (D) Double immunohistochemistry for Notch1 and c-fos shows that the faintly Notch1-labeled MCs, in the Notch1cKOKln, appear c-fos-negative. ${ }^{*} P<0.05$; error bars are SEM. WT, wildtype; GCL, granule cell layer; GL, glomerulus; MCL, Mitral cell layer. Scale bars, $25 \mu \mathrm{m}$ (A and D), $100 \mu \mathrm{m}(\mathrm{C})$.

affected. Indeed, we observed a $30 \%$ decrease in spontaneous firing rate in the Notch1cKOKlns as compared to wildtype mice $\left(6.2 \pm 0.78\right.$ and $8.8 \pm 0.21 \quad$ spikes $/ \mathrm{s} ; \quad t_{4}=3.250, \quad P=0.031$ $n=110$ cells in wildtype and $n=130$ cells in knockouts; Fig. 6E). The baseline activity of the wildtype MCs is in line with a previous report using extracellular recordings in ketamine-anesthetised rat OBs (Nagayama et al., 2004) and mouse OBs (Davison \& Katz, 2007). Based on the evidence that c-fos expression, as a readout of neural activity, was considerably higher in the GCL, we addressed whether synchrony, which may be achieved by the inhibitory drive of GC interneurons onto MCs (Schoppa, 2006), was altered. Crosscorrelation analysis, as described before (Abeles, 1982), was carried out between all cell pairs in the recordings from wildtypes and Notch1cKOKlns. We observed that amyl acetate exposure increased the synchronous firing of wildtype MCs of the dorsal OB as compared to the stimulation with benzaldehyde $(56 \% \pm 11$ vs. $32 \% \pm 10 ; F_{1,8}=19.40, P=0.002$; Fig. $6 \mathrm{~F}$; grey boxes, synchronous interaction; white boxes, no synchronous interaction). On the other hand, Notch1cKOKln MCs appear to be highly synchronised independently of the stimulus $(81 \% \pm 20$ vs. $70 \% \pm 28$; $F_{1,8}=2.75, P=0.135$; Fig. $6 \mathrm{~F}$ ). To further investigate whether the increase in synchrony may result from stronger feedback inhibition from granule cells, we analysed the cumulative activity of all the recorded cells $2.5 \mathrm{~s}$ before and $2.5 \mathrm{~s}$ after the stimulus in wildtypes and knockouts. We observed, as previously reported (Davison \& Katz, 2007), that MC activities were either induced or suppressed in response to odour stimulation (Fig. 7A). We observed that, in wildtypes, the majority of the neurons increase their firing frequency after stimulus application $(60 \% \pm 7)$, whereas in the Notch1cKOs the majority of cells displayed a reduction in firing rate $(70 \% \pm 7$; $F_{1,8}=20.7, P=0.002$; Fig. 7B). Computing the average cumulative frequencies of all cells across all animals per genotype, we observe that wildtypes, in contrast to Notch1cKOs, showed a slight net increase in firing frequencies upon the stimulus $(+20 \% \pm 5$ vs. $-12 \% \pm 9 ; t_{4}=2.7, P=0.017$; Fig. 7C).

We conclude that loss of Notch1 in MCs has profound consequences on MC physiology, a loss that seems to affect OB network function and specific responses to sensory stimulation.

\section{Notch1 influences odour avoidance but not attraction to novel odour}

Our data indicate that Notch1 may contribute to olfactory processing. We therefore addressed whether olfactory behaviour was altered in the Notch1cKOKln mice. We took advantage of three simple one-trial paradigms to test olfactory behaviour. The first experiment tests the discrimination of a novel odour, Swiss cheese, from a familiar odour, the bedding (Fig. 8E). Independently of the genotype, the majority of the mice found the cheese within a 5-min window. Only two mice per genotype failed to find the bait. This indicates that olfactory perception of a novel odour is unaffected in the Notch1cKOKlns (Fig. 8A; $t_{16}=0.1667, P=0.870,2.1 \pm 0.6$ vs. $1.9 \pm 0.7 \mathrm{~min} ; n=9$ per genotype). Thereafter, we tested the olfactory attraction to another novel odour, vanilla, vs. a neutral odour, water (Fig. 8F). In both wildtype and Notch1cKO mice, vanilla is slightly attractive as compared to water, although based on 
A

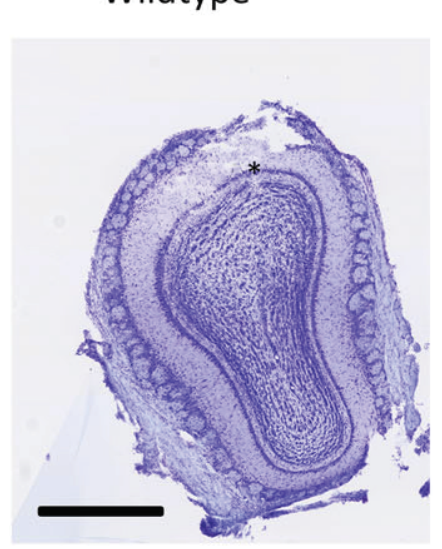

B

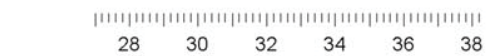

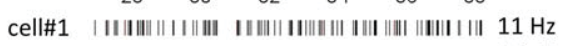
cell\#2 | ||||||||||||| | | |||| | | || || || || | $3.4 \mathrm{~Hz}$ cell\#3 |||||| ||||||| ||||| || || |||| || | || || | | || || || || || || || || | $10 \mathrm{~Hz}$ cel|\#4 I|| | | | | | | ||| || || || || || || || || || | | || || || || || || | || || || || ||| $10 \mathrm{~Hz}$ cell\#5 || | || || ||| | | ||| || ||l||| || | | | $0.5 \mathrm{~Hz}$ Odor

C

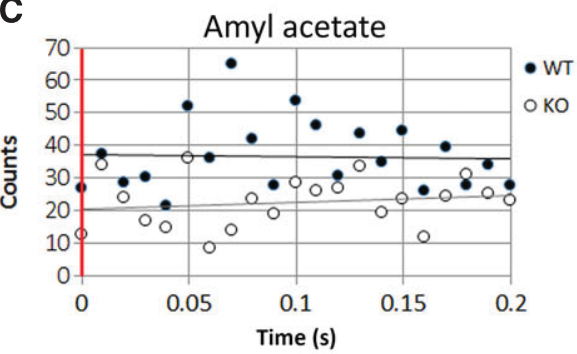

D

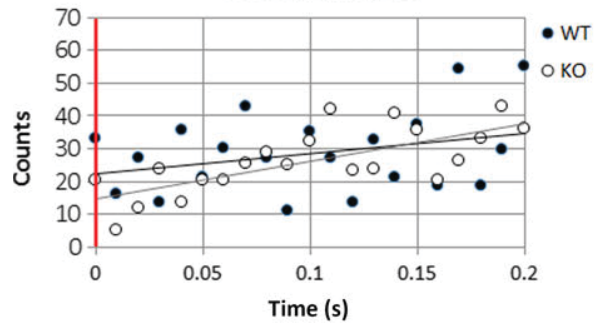

E

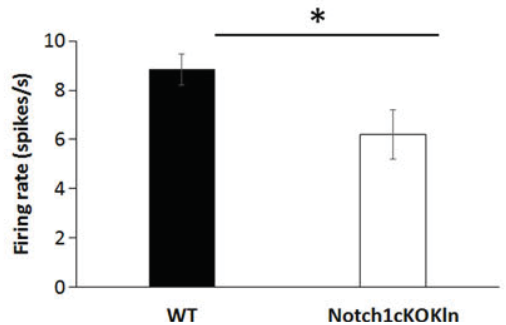

Notch1cKOKIn

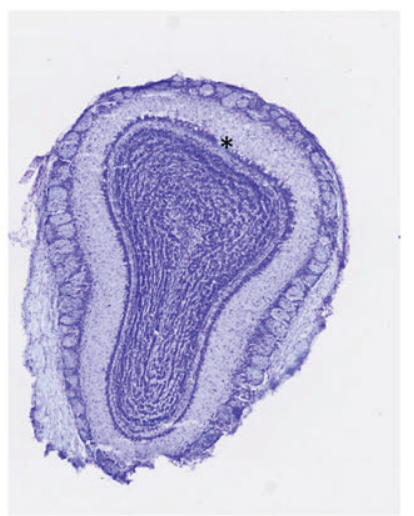

|

$\begin{array}{llllll}28 & 30 & 32 & 34 & 36 & 38\end{array}$

| | || || || ||||||||||| || | || | || || || || | || || || || $13 \mathrm{~Hz}$

|| || || || || || || || | | ||| | || || ||| || $4.8 \mathrm{~Hz}$

$\|+\|\|+\||+|||||\|\|||||\|||\||||| 5.6 \mathrm{~Hz}$

| | | || |||| || | | || || || ||| | || $4 \mathrm{~Hz}$

|||\|\|| \|\|\|\|||||||$|\|||||| \mid 4 \mathrm{~Hz}$

$\mathbf{C}^{\prime}$

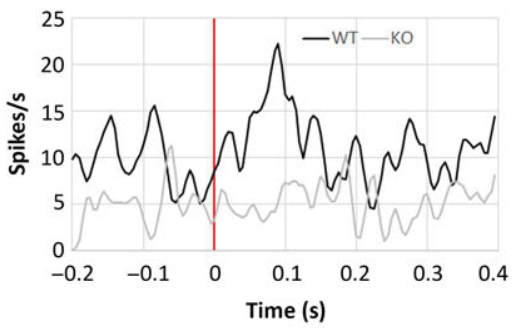

$D^{\prime}$
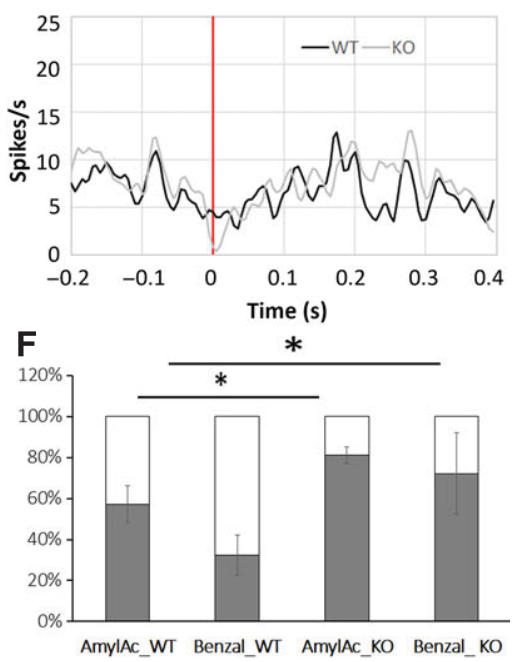

FIG. 6. Notch1 regulates the response of MCs to odours. (A) Nissl staining showing the electrode penetration sites (black stars) in the MCL of the dorsal OB of wildtype and Notch1cKOKln mice. (B) Example of raster plots of five sorted neurons for one electrode site in one wildtype and one Notch1CKOKln mouse. Red bars indicates the odour stimulus of $1 \mathrm{~s}$. (C) Scattered dot plot showing the peristimulus response (bin $=10 \mathrm{~ms}$ ) to the amyl acetate trigger (red vertical line) indicates that the spike counts in response to the stimulus are significantly lower in the NotchlcKOKln than the wildtype. Trend line for wildtype is in blue and for $\mathrm{KO}$ in magenta. $\left(\mathrm{C}^{\prime}\right)$ A peristimulus time histogram (bin $=5 \mathrm{~ms}$ ) indicates that the wildtypes' firing rate in response to the amyl acetate stimulus (red vertical line) is significantly larger than that of the Notch1cKOs (D) Scattered plot showing the peristimulus (bin $=10$ ms) response to the benzaldehyde trigger shows no difference between wildtypes and Notch1cKOs. $\left(\mathrm{D}^{\prime}\right)$ A peristimulus time histogram (bin $=5 \mathrm{~ms}$ ) shows no change in the firing rate to benzaldheyde in either genotype. (E) Chart representing the spontaneous firing rate of wildtype and knockout MCs indicates a reduced firing rate in Notch1cKOKln as compared to wildtype. (F) Cumulative percentage histogram represents the proportion of synchronous firing neurons (grey boxes) and neurons that display no interaction (white boxes). In the wildtypes following exposure to amyl acetate the proportion of synchronous firing neurons is increased compared to benzaldehyde. In the knockouts the majority of the neurons fire synchronously (grey boxes) and there is no difference between amyl acetate and benzaldehyde. WT, wildtype. ${ }^{*} P<0.05$. Error bars are SEM. Scale bar in A, $1 \mathrm{~mm}$. 
A

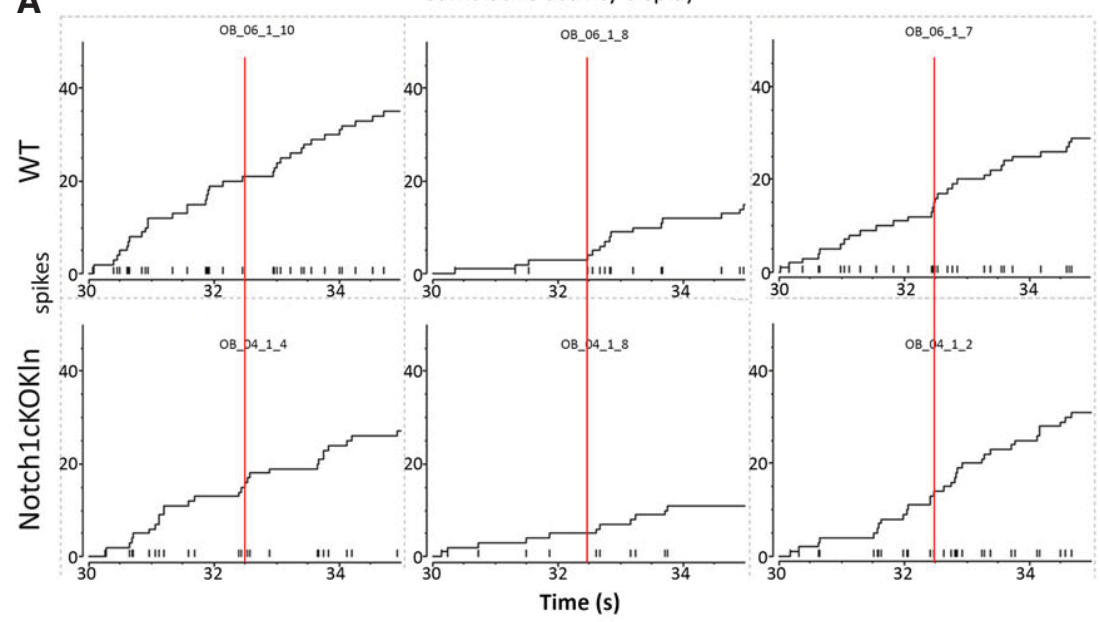

$\mathbf{B}_{100 \%}$

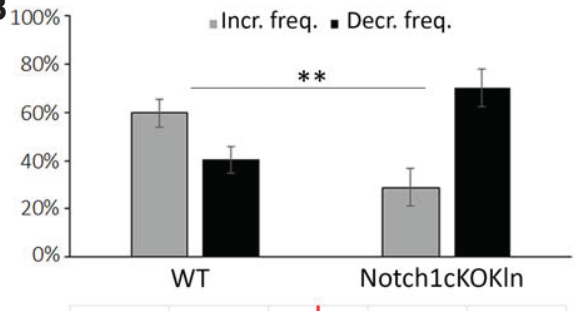

C

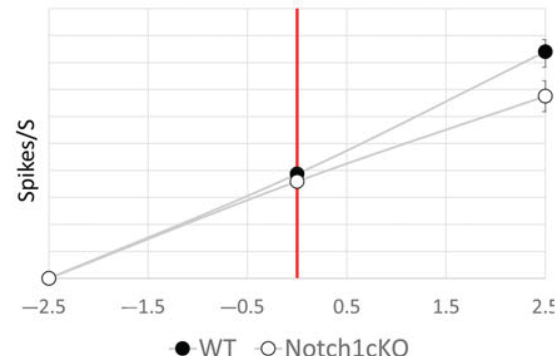

FIG. 7. Notch1cKOkln MCs display increased signal inhibition upon amyl acetate. (A) Representative cumulative activity plots for three cells in a wildtype and Notch1cKOKln show how the firing response of each cell develops before and after the odour (red line). (B) Chart summarising the differential distribution of neurons with increased or decreased frequency in response to the odour in wildtypes and Notch1cKOKlns. (C) Chart summarising the average cumulative frequencies for wildtypes and Notch $1 \mathrm{cKOK}$ lns $2.5 \mathrm{~s}$ before and after the odour presentation. Each horizontal line indicates 10 spikes/s. $* P<0.05, * * P<0.01$. Error bars are SEM.
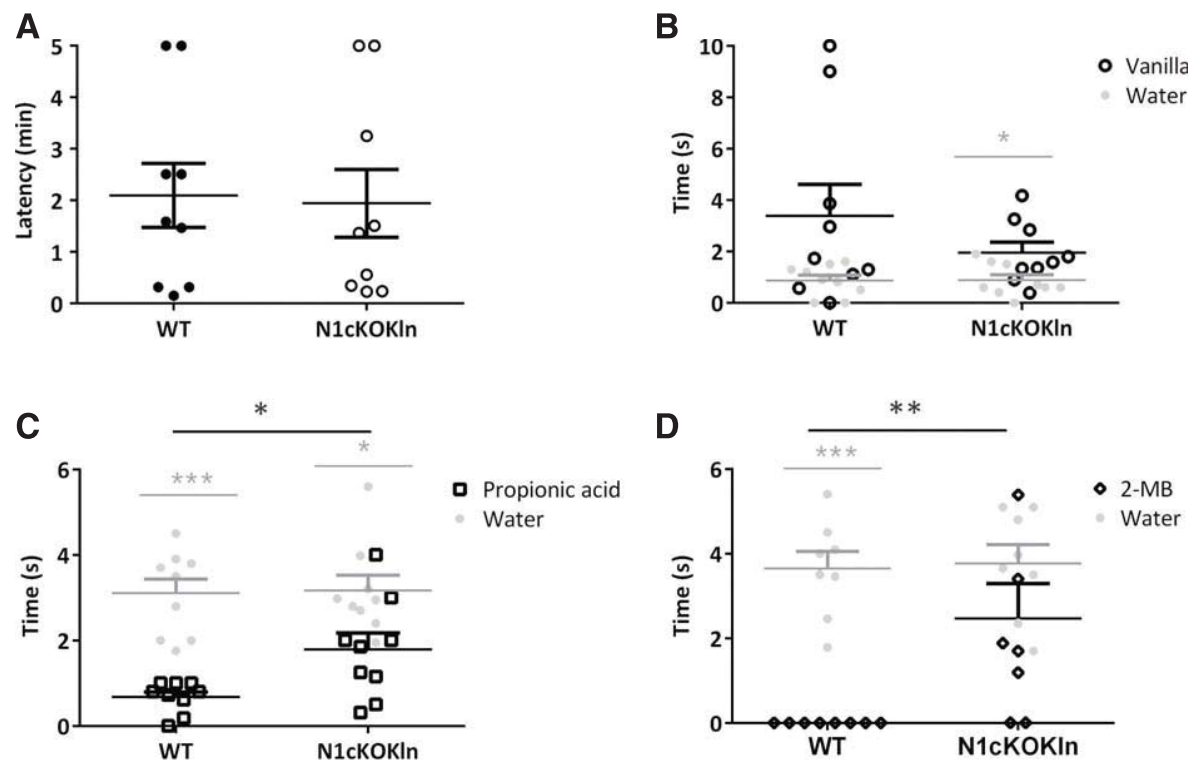

$\mathbf{E}$

$\mathbf{F}$

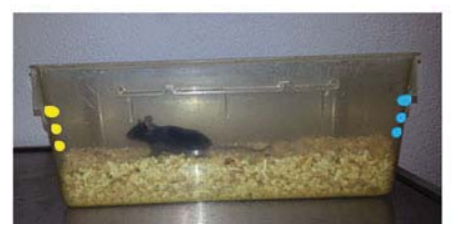

FIG. 8. Olfactory avoidance is impaired in the Notch1cKOKln mice. (A) Notch1cKOKlns spend the same amount of time as wildtypes to find a novel odour (Swiss cheese). (B) Notch1cKOKlns spend the same time with a novel odour (vanilla) as wildtype. Notch1cKOklns have significant preference for vanilla over water. (C) Olfactory avoidance, after propionic acid exposure, is significantly reduced in Notch1cKOKlns as compared to wildtype. In both genotypes mice spend more time sniffing water. (D) Olfactory avoidance to 2-MB acid exposure is significantly decreased in N1cKOKln mice as compared to wildtypes. Only wildtype mice spend more time sniffing water. (E and F) Representative photo for measuring (E) latency to Swiss cheese (orange dice) and (F) time sniffing vanilla, propionic acid or 2-MB acid (yellow drops) as opposed to water (blue drops). WT, wildtype. In B-D, significant behavioural differences between genotypes are indicated by black horizontal bars and asterisks and significant difference in sniffing times between the odour and water within a genotype are indicated by grey horizontal bars and asterisks. ${ }^{*} P<0.05,{ }^{*} P<0.01$ and $* * * P<0.001$. Error bars are SEM. 
the variability of the sampling it appears below significance in wildtypes (Fig. 8B; wildtypes, $t_{16}=2.040, P=0.058,3.4 \pm 1.2$ vs. $0.8 \pm 0.2 \mathrm{~s} ; \quad$ knockouts, $t_{16}=2.36, \quad P=0.031,2.0 \pm 0.4$ vs. $0.9 \pm 0.2 \mathrm{~s} ; n=9$ per odour). Mice of the two genotypes displayed comparable sniffing times; nevertheless, the Notch1cKOKlns had a tendency to sniff less than the wildtype controls (Fig. 8B; $t_{16}=1.114, P=0.281,3.4 \pm 1.2$ vs. $2.0 \pm 0.4 \mathrm{~s} ; n=9$ per genotype). We next examined whether avoidance of two unpleasant acidic odours, propionic acid and 2-methylbutyric acid (2-MB), is affected in the Notch1cKOKlns (Fig. 8F). Acids can rapidly trigger an innate motor reflex (Kobayakawa et al., 2007). Wildtypes and Notch1cKOKln display a differential avoidance response to propionic acid as compared to water (Fig. 8C; wildtypes, $t_{16}=6.878$, $P<0.001, \quad 0.7 \pm 0.1$ vs. $3.1 \pm 0.3 \mathrm{~s} ; \quad$ knockouts, $t_{16}=2.62$, $P=0.018,1.8 \pm 0.4$ vs. $3.2 \pm 0.4 \mathrm{~s} ; n=9$ per odour). Nevertheless, we observed a significant delay in the avoidance response in Notch1cKOKln mice as compared to wildtype controls after propionic acid (Fig. 8C; $t_{16}=2.707, \quad P=0.016, \quad 0.7 \pm 0.1 \quad$ vs. $1.8 \pm 0.4 \mathrm{~s} ; n=9$ per genotype). The defect in avoidance of the Notch1cKOklns was even more apparent in response to $2-\mathrm{MB}$ as compared to water (Fig. 8D; wildtypes, $t_{14}=9.08, \quad P<0.001$, $0.0 \pm 0.0$ vs. $3.7 \pm 0.4 \mathrm{~s} ; \quad$ knockouts, $t_{14}=1.47, \quad P=0.187$, $2.5 \pm 0.8$ vs. $3.7 \pm 0.4 \mathrm{~s} ; n=8$ per odour). As a result, Notch1cKOKlns displayed significantly less avoidance to 2-MB exposure than did wildtypes (Fig. 8D; $t_{14}=3.0, \quad P=0.010, \quad 0.0 \pm 0.0 \quad$ vs. $2.5 \pm 0.8 \mathrm{~s} ; n=8$ per genotype). This indicates that Notch1 loss affected olfactory avoidance of an unpleasant acidic odour but had no effect on the perception of novel odours.

\section{Discussion}

In this study we show that, in the mouse olfactory bulb, Notch signalling is odour-dependent and is localised mainly in MCs of the OB. Loss of function of Notch1 in this layer results in ectopic neural activity in the adjacent GCL. In addition, loss of Notch1 compromises the ability of MCs to respond to specific odours, altering synchronous firing in basal conditions. At the behavioural level, we observed that Notch1 loss affects olfactory avoidance but not olfactory preference to a novel odour. These results indicate that Notch1 is implicated in MCs physiology and olfactory behaviour.

\section{Notch signalling components are present in the adult $O B$}

Our immunohistochemical analysis has revealed that Notch1 is expressed abundantly in the adult $\mathrm{OB}$, with the strongest expression in MCs. These are the only principal cells of the $\mathrm{OB}$ receiving direct input from the olfactory receptor neurons and relaying the olfactory signal to cortical structures.

Interestingly, we observed that Jagged1, the cognate ligand of Notch1, is expressed in the olfactory nerve layer, juxtaposed to Notch1 on MCs. This expression pattern would grant signalling directionality from olfactory sensory neurons to MCs, along with the flow of olfactory transmission.

Jagged1 is also co-expressed with Notch1 in MCs. This arrangement is in contrast to the classical dogma of Notch signalling where the signalling cell expresses the ligand and the receiving cells the receptor (D'Souza et al., 2008). Nevertheless, MCs also express Reelin and the Reelin receptor, ApoER2, and appear to have unique autocrine functions (Herrmann et al., 2008). In addition, the presence of Notch1 and Jagged1 in the 'cis' configuration in MCs may represent a strategy for dampening the signal (D'Souza et al., 2008). We have also detected Jagged1 in resident glial cells. It is now established that glia feed back onto neurons to regulate synaptic plasticity (Eroglu \& Barres, 2010). Based on this result and the evidence indicating that Jagged1 is expressed in the mature brain (Stump et al., 2002), and is relevant for neuronal physiology (Sargin et al., 2013), it is likely that neuronal- or glial-derived Jagged1 may influence the activity of Notch1-positive neurons in the OB.

It appears, therefore, that Notch pathway components are expressed in specific cellular subsets of the $\mathrm{OB}$ and may be involved in olfactory signalling.

\section{Odour stimulation triggers Notch transcriptional activity}

Taking advantage of a Notch transcriptional responsive element (Mizutani et al., 2007), which is inverted in the direction of transcription upon CamKII- $\alpha:$ :cre recombination (Gradinaru et al., 2008), we have observed that Notch signalling is induced specifically in MCs and their projections following odour exposure. These results suggest that, similarly to the Notch-DSL signalling in the Drosophila olfactory system (Lieber et al., 2011), odorant stimulation triggers Notch activity. The dependence of Notch signalling on synaptic activity has been observed in other neuronal ensembles such as the rodent hippocampus (Alberi et al., 2011) and the neuromuscular synapses in Drosophila (de Bivort et al., 2009). In our study, in response to amyl acetate, Notch-dependent YFP activity was visible in ensembles of MCs of the dorsal $\mathrm{OB}$ and clusters of glomeruli on the dorsal and ventral OB. This activation pattern is characteristic of amyl acetate (Guthrie et al., 1993; Xu et al., 2003), although with our transcriptional YFP reporter we have no information on the degree of activation. Therefore, lateral glomeruli, whose activation is weaker (Guthrie et al., 1993), still appear YFP-positive. In addition, only clusters of dorsal MCs are visible. This can be explained by the specific tagged YFP reporter which localises preferentially at the distal dendritic membranes (Mattis et al., 2012). Only if MCs are strongly activated does YFP accumulate in their cytoplasm. Thereby, Notch1 appears the relevant signalling receptor in the principal cells of the $\mathrm{OB}$, similarly to its requirement for hippocampal plasticity (Wang et al., 2004; Alberi et al., 2011). On the whole, we conclude that odour stimulation is required to trigger Notch transcriptional activity, supporting a role for Notch1 in olfactory transmission.

\section{Notch1 regulates $M C$ activity}

To further investigate whether Notch1 has a functional role in olfactory activity, we took advantage of our newly generated mouse model in which Notch1 is ablated in mature neurons of the forebrain. According to the observed expression pattern of CamKII- $\alpha$ in the olfactory bulb in our and other studies (Liu, 2000; Kato et al., 2013), the Notch1cKOKln (CamKII- $\alpha:$ : cre) mouse displays significantly lower immunoreactivity for Notch1 in MCs. Expression of Notch1 may be affected in other layers of the OB-expressing CamKII- $\alpha$ (Zou et al., 2002; Néant-Fery et al., 2012). Nevertheless, we believe that, based on the robust expression of both CamKII- $\alpha$ and Notch1 in MCs, we have achieved functional deletion of Notch1 in this cell population of the OB. Ablation of Notch1 expression in this mouse line was homogeneous across the MC population and progressive with age. This suggests that, in the conditional knockouts for Notch1, the dosage of the cre recombinase is a critical factor (Gaiano et al., 2012; Zheng et al., 2012). Nevertheless, partial lossof-function mouse models for Notch1 have been previously used and have indicated that levels of Notch1 may be critical for neuronal plasticity and information processing (Costa et al., 2003; Wang 
et al., 2004). Western blot analysis from olfactory bulb lysates confirmed that the levels of Notch1 was significantly lower in the Notch1cKOKlns than in controls but not completely abolished. This suggests that Notch1 expression is maintained in other populations of the bulb as well as in migrating neuroblasts of the peduncle subventricular zone, where Notch1 activity is strongest (Basak et al., 2012; and data not shown). We observed that, as a result of the consistent loss of Notch1 in MCs, their baseline activity was reduced. In mice, spontaneous activity in this cell population is intrinsic to the availability of NMDAR1 (Heinbockel et al., 2004) and depends on corticofugal innervation (Zhan \& Heinbockel, 2013). Interestingly, loss of Notch1 in pyramidal cells of the hippocampus causes an NMDAR1KO-like phenotype (Alberi et al., 2011). It is possible that, similarly to the hippocampus, loss of Notch1 may also affect the excitability of principal cells in the OB.

On the other hand, stimulus-evoked responses are multifactorial and depend on the excitability of MCs, olfactory input mediated by olfactory receptor neurons and the reciprocal inhibition from GCs. We observed that dorsal MCs, which in wildtype mice respond to the specific odour amyl acetate (Xu et al., 2003), fail to do so in the absence of Notch1. Nevertheless, dorsal MCs from both Notch1cKOKln and wildtypes lack any response to benzaldheyde, as expected by its odorant map (Matsumoto et al., 2010). This result indicates that the odour-evoked response of MCs is significantly affected in Notch1cKOKln mice. We further addressed whether increased lateral inhibition may underlie this defect. Along these lines, we observed that cage control Notch1cKOkln mice display ectopic activity in GCs, as indicated by c-fos expression. Cage control mice, which are exposed to familiar odours of their housing, normally show low levels of neuronal activity in the GCL, as a result of olfactory adaptation of MCs (Davison \& Katz, 2007) and the intrinsic hyperpolarised state of GCs (Labarrera et al., 2013). Based on the reduced spontaneous activity of MCs in anesthetised mice, it is unlikely that altered GCs activity depends on the tone of the input cells, but rather suggests that, in the Notch1cKOkln model, GCs activity may be altered in a non-cell-autonomous manner. Non-cell-autonomous effects on the development of GCs inhibitory synapses have previously been observed in Tbr2cKO mice, where Tbr2 expression is specifically deleted in MCs (Mizuguchi et al., 2012). Indeed, there is increasing evidence that MCs activity can contribute to the maturation of GCs synapses (Kelsch et al., 2012; Breton-Provencher et al., 2014), and a differential repertoire of glutamate receptors at dendrodendritic synapses can significantly affect the properties of these cells (Abraham et al., 2010).

To further investigate the effects of lateral inhibition in Notch1cKOkln, we analysed the odour-evoked responses of the recorded cells to amyl acetate. We observed that, in the knockouts, the majority of neurons decreased their firing activity in contrast to wildtypes. The net result is that, in response to odour, MCs of the Notch1cKOkln do not show the characteristic increase in firing upon amyl acetate (Tan et al., 2010). To further address the properties of neighbouring MCs we investigated odour-evoked synchrony. Correlated firing activity, upon odour stimulation, has been reported in studies using extracellular recordings in several animal models from Drosophila (Kazama \& Wilson, 2009) to rodents (Buonviso \& Chaput, 1990; Kashiwadani et al., 1999; David et al., 2009). In addition, synchrony appears to be largely mediated by GCs feedback inhibition onto MCs (Schoppa \& Westbrook, 2001; Schoppa, 2006; Abraham et al., 2010) as well as gap junctions between MCs (Christie et al., 2005). We observed that amyl acetate produces an increase in synchrony between neighbouring MCs in the wildtype $\mathrm{OB}$ as compared to benzaldehyde exposure. On the other hand, in MCs of the Notch1cKOKlns, synchrony is significantly higher $(80 \%$ vs. $56 \%)$ than in wildtypes and appears to be independent of the odorant applied. The high synchrony achieved in the absence of Notch1 may result from the observed increased in GCs baseline activity though this needs to be confirmed by patch-clamp recordings in OB slices. In addition, stronger synchrony of MCs in the Notch1cKOKln mice may represent a compensation mechanism to the reduced firing responses which warrants signal propagation from the periphery to central structures. In future studies maturation of reciprocal synapses in the Notch1cKOKln will be investigated in order to resolve cell-autonomous from non-cell-autonomous effects of Notch1 loss in the OB and will offer new insight in the role of Notch in the communication between MCs and GCs.
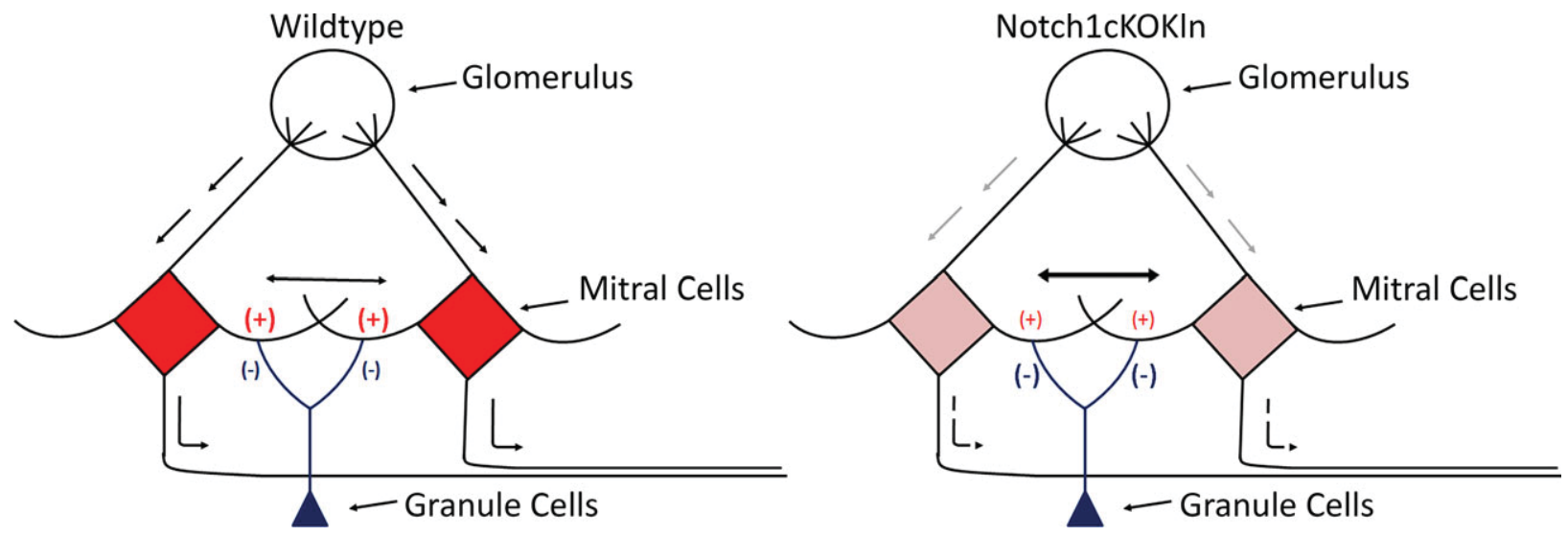

FIG. 9. Schematic illustration of the effect of Notch1 loss on olfactory processing. Notch1-positive MCs (red) project their primary dendrites to a glomerulus, their long axon to cortical structures. Their secondary dendrites are contacted by reciprocal synapses of granule cells (blue); in addition, MCs are connected via gap junctions (mitral neurites junction). In wildtypes, when an odour is applied, odorant input is transmitted to MCs (descending arrows) and Notch activity is induced. In response to the strong depolarisation, MCs activate (bold positive signs) GCs which, in turn, by feedback inhibition (light negative signs), synchronise MC firing (bidirectional arrow) and determine signal discrimination and olfactory behaviour (arrows along the MC axon). When Notch1 expression is strongly reduced (pale red), as in the Notch1cKOKln, the spontaneous as well as the evoked activity of MCs is reduced (light positive signs). In the same model, feedback inhibition is increased (negative signs) leading to an increase in synchronous firing (thicker bidirectional arrow) and damping the odour-evoked responses. This may result in a compensatory effect on the outgoing signal which can lead to a partial olfactory defect (broken arrows along the MCs axon). 


\section{Notch1 loss affects olfactory avoidance but not olfactory perception}

To investigate whether the loss of Notch1 in the OB results in altered olfactory behaviour, we performed four single-trial olfactory paradigms. No difference in olfactory attraction to the novel odours, Swiss cheese and vanilla, was observed between the genotypes. This is in agreement with the normal exploratory olfactory performance of the aged male RBPJKcKO mice (Sato et al., 2012). Nevertheless, in our study we did not observe an olfactory preference deficit as displayed in the RBPJKcKO mice (Sato et al., 2012). This could be due to on the type of paradigm used or the difference in age between the Notch1cKOKln and the RBPJKcKO mice. On the other hand, the Notch1cKOKlns displayed a slower reaction in the avoidance test to propionic acid and 2-MB acid. Acids in high concentration have a pungent smell with a low threshold for sensitivity that causes an avoidance reflex in the mouse within seconds (Kobayakawa et al., 2007). Deficit in innate flight responses to predator (trimethyl thiazoline; TMT) or pungent (acids) odours have been shown in mutant mice for olfactory sensory neurons (Kobayakawa et al., 2007; Bepari et al., 2012; Cao et al., 2012). TMT or aliphatic acids, such as propionic and $2-\mathrm{MB}$, can specifically activate the dorsal glomeruli. This signal is then relayed through MCs, olfactory cortex and striatum to the hypothalamic pituitary axis, triggering the avoidance response (Kobayakawa et al., 2007). One possibility is that loss of Notch1 in MCs or olfactory interneurons impairs signal transmission to these centres. On the other hand, it remains to be investigated whether loss of Notch1 in the basal hypothalamus, as expected in this cre line (Minichiello et al., 1999), affects the physiology of the hypothalamic pituitary axis.

On the whole, our study shows an important, yet undiscovered, role for Notch1 in olfactory activity with apparent cell-autonomous and non-cell-autonomous effects on MCs and GCs, respectively (Fig. 9). In addition, loss of Notch1 affects olfactory avoidance but not perception of novel odours. As this pathway appears to be affected during aging and in several neuronal pathologies (Alberi et al., 2013), it is likely that the olfactory deficit associated with these conditions is also the result of Notch signalling imbalances. More studies in the future will address how Notch1 activity in MCs influences OB network activity and olfactory encoding.

\section{Acknowledgements}

We would like to thank Marguerite Kaczorowski for her continuous technical support throughout the project. We are grateful to Nicholas Gaiano for sharing the multimeric Notch-responsive element constructs with us and for all his support during the years. We thank Professor Klein for sharing the CamKII- $\alpha$ :cre mouse line with us. We are grateful to Professor Radtke for sharing the Notch1 floxed mouse lines with us. We would like to thank Alexandre Babalian for his technical support for the extracellular recordings. We would like to thank Professor Falquet for his assistance in the statistical analysis. This work is supported by the Swiss National Foundation and Synapsis Foundation Switzerland.

\section{Abbreviations}

EPL, external plexiform layer; GC, granule cell; GCL, granule cell layer; MC, mitral cell; MCL, mitral cell layer; NICD1, Notch1 in the Notch intracellular domain; OB, olfactory bulb; PGC, periglomerular cell; PGL, periglomerular layer; YFP, yellow-fluorescent protein.

\section{References}

Abeles, M. (Ed) (1982) Interactions between pairs of cells. In Local Cortical Circuits, Studies of Brain Function. Springer, Berlin, pp. 27-39.
Abraham, N.M., Egger, V., Shimshek, D.R., Renden, R., Fukunaga, I., Sprengel, R., Seeburg, P.H., Klugmann, M., Margrie, T.W., Schaefer, A.T. \& Kuner, T. (2010) Synaptic inhibition in the olfactory bulb accelerates odor discrimination in mice. Neuron, 65, 399-411.

Alberi, L., Liu, S., Wang, Y., Badie, R., Smith-Hicks, C., Wu, J., Pierfelice, T.J., Abazyan, B., Mattson, M.P., Kuhl, D., Pletnikov, M., Worley, P.F. \& Gaiano, N. (2011) Activity-induced Notch signaling in neurons requires Arc/Arg3.1 and is essential for synaptic plasticity in hippocampal networks. Neuron, 69, 437-444.

Alberi, L., Hoey, S.E., Brai, E., Scotti, A.L. \& Marathe, S. (2013) Notch signaling in the brain: In good and bad times. Ageing Res. Rev., 12, 801814.

Albéri, L., Lintas, A., Kretz, R., Schwaller, B. \& Villa, A.E.P. (2013) The calcium-binding protein parvalbumin modulates the firing 1 properties of the reticular thalamic nucleus bursting neurons. J. Neurophysiol., 109, 2827-2841.

Artavanis-Tsakonas, S., Rand, M.D. \& Lake, R.J. (1999) Notch signaling: cell fate control and signal integration in development. Science, 284, 770-776.

Aschauer, D.F., Kreuz, S. \& Rumpel, S. (2013) Analysis of transduction efficiency, tropism and axonal transport of AAV serotypes 1, 2, 5, 6, 8 and 9 in the mouse brain. PLoS One, 8, e76310.

Basak, O., Giachino, C., Fiorini, E., Macdonald, H.R. \& Taylor, V. (2012) Neurogenic subventricular zone stem/progenitor cells are Notch1dependent in their active but not quiescent state. J. Neurosci., 32, 5654-5666.

Bepari, A.K., Watanabe, K., Yamaguchi, M., Tamamaki, N. \& Takebayashi, H. (2012) Visualization of odor-induced neuronal activity by immediate early gene expression. BMC Neurosci., 13, 140.

Berezovska, O., Xia, M.Q. \& Hyman, B.T. (1998) Notch is expressed in adult brain, is coexpressed with presenilin-1, and is altered in Alzheimer disease. J. Neuropath. Exp. Neur., 57, 738-745.

de Bivort, B.L., Guo, H.-F. \& Zhong, Y. (2009) Notch signaling is required for activity-dependent synaptic plasticity at the Drosophila neuromuscular junction. J. Neurogenet., 23, 395-404.

Breton-Provencher, V., Coté, D. \& Saghatelyan, A. (2014) Activity of the principal cells of the olfactory bulb promotes a structural dynamic on the distal dendrites of immature adult-born granule cells via activation of NMDA receptors. J. Neurosci., 34, 1748-1759.

Buonviso, N. \& Chaput, M.A. (1990) Response similarity to odors in olfactory bulb output cells presumed to be connected to the same glomerulus: electrophysiological study using simultaneous single-unit recordings. $J$. Neurophysiol., 63, 447-454.

Cao, L., Schrank, B.R., Rodriguez, S., Benz, E.G., Moulia, T.W., Rickenbacher, G.T., Gomez, A.C., Levites, Y., Edwards, S.R., Golde, T.E., Hyman, B.T., Barnea, G. \& Albers, M.W. (2012) A $\beta$ alters the connectivity of olfactory neurons in the absence of amyloid plaques in vivo. Nat. Commun., 3, 1009.

Christie, J.M., Bark, C., Hormuzdi, S.G., Helbig, I., Monyer, H. \& Westbrook, G.L. (2005) Connexin36 mediates spike synchrony in olfactory bulb glomeruli. Neuron, 46, 761-772.

Costa, R.M., Honjo, T. \& Silva, A.J. (2003) Learning and memory deficits in Notch mutant mice. Curr. Biol., 13, 1348-1354.

David, F.O., Hugues, E., Cenier, T., Fourcaud-Trocmé, N. \& Buonviso, N. (2009) Specific entrainment of mitral cells during gamma oscillation in the rat olfactory bulb. PLoS Comput. Biol., 5, e1000551.

Davison, I.G. \& Katz, L.C. (2007) Sparse and selective odor coding by mitral/ tufted neurons in the main olfactory bulb. J. Neurosci., 27, 2091-2101.

D'Souza, B., Miyamoto, A. \& Weinmaster, G. (2008) The many facets of Notch ligands. Oncogene, 27, 5148-5167.

Eroglu, C. \& Barres, B.A. (2010) Regulation of synaptic connectivity by glia. Nature, 468, 223-231.

Gaiano, N., Alberi, L. \& Liu, S. (2012) Expression of Notch proteins in pyramidal neurons in vivo. J. Biol. Chem., 287, 24595.

Gradinaru, V., Thompson, K.R. \& Deisseroth, K. (2008) eNpHR: a Natronomonas halorhodopsin enhanced for optogenetic applications. Brain Cell Biol., 36, 129-139.

Guthrie, K.M., Anderson, A.J., Leon, M. \& Gall, C. (1993) Odor-induced increases in c-fos mRNA expression reveal an anatomical "unit" for odor processing in olfactory bulb. Proc. Natl. Acad. Sci. USA, 90, 3329-3333.

Hack, I., Bancila, M., Loulier, K., Carroll, P. \& Cremer, H. (2002) Reelin is a detachment signal in tangential chain-migration during postnatal neurogenesis. Nat. Neurosci., 5, 939-945.

Heinbockel, T., Heyward, P., Conquet, F. \& Ennis, M. (2004) Regulation of main olfactory bulb mitral cell excitability by metabotropic glutamate receptor mGluR1. J. Neurophysiol., 92, 3085-3096. 
Hellwig, S., Hack, I., Zucker, B., Brunne, B. \& Junghans, D. (2012) Reelin together with ApoER2 regulates interneuron migration in the olfactory bulb. PLoS One, 7, e50646.

Herrmann, G., Mishev, G. \& Scotti, A.L. (2008) Olfactory bulb interneurons releasing NO exhibit the Reelin receptor ApoEr2 and part of those targeted by NO express Reelin. J. Chem. Neuroanat., 36, 160-169.

Ilagan, M.X.G., Lim, S., Fulbright, M., Piwnica-Worms, D. \& Kopan, R. (2011) Real-time imaging of Notch activation using a Luciferase Complementation-based Reporter. Sci. Signal., 4, rs7.

Kashiwadani, H., Sasaki, Y.F., Uchida, N. \& Mori, K. (1999) Synchronized oscillatory discharges of mitral/tufted cells with different molecular receptive ranges in the rabbit olfactory bulb. J. Neurophysiol., 82, 1786-1792.

Kato, H.K., Gillet, S.N., Peters, A.J., Isaacson, J.S. \& Komiyama, T. (2013) Parvalbumin-expressing interneurons linearly control olfactory bulb output. Neuron, 80, 1218-1231.

Kaupp, U.B. (2010) Olfactory signalling in vertebrates and insects: differences and commonalities. Nat. Rev. Neurosci., 11, 188-200.

Kazama, H. \& Wilson, R.I. (2009) Origins of correlated activity in an olfactory circuit. Nat. Neurosci., 12, 1136-1144.

Kelsch, W., Li, Z., Eliava, M., Goengrich, C. \& Monyer, H. (2012) GluN2B-containing NMDA receptors promote wiring of adult-born neurons into olfactory bulb circuits. J. Neurosci., 32, 12603-12611.

Kobayakawa, K., Kobayakawa, R., Matsumoto, H., Oka, Y., Imai, T., Ikawa, M., Okabe, M., Ikeda, T., Itohara, S., Kikusui, T., Mori, K. \& Sakano, H. (2007) Innate versus learned odour processing in the mouse olfactory bulb. Nature, 450, 503-508

Labarrera, C., London, M. \& Angelo, K. (2013) Tonic inhibition sets the state of excitability in olfactory bulb granule cells. J. Physiol., 591, 18411850 .

Lieber, T., Kidd, S. \& Struhl, G. (2011) DSL-Notch signaling in the Drosophila brain in response to olfactory stimulation. Neuron, 69, 468-481.

Liu, N. (2000) Regional distribution of protein kinases in normal and odordeprived mouse olfactory bulbs. Chem. Senses, 25, 401-406.

Markopoulos, F., Neubauer, F.B., Berger, T. \& Scotti, A.L. (2008) Reassembling a system from the sensor to cerebral representation: the olfactory system in vitro. Neuroscience, 156, 1048-1063.

Matsumoto, H., Kobayakawa, K., Kobayakawa, R., Tashiro, T., Mori, K., Sakano, H. \& Mori, K. (2010) Spatial arrangement of glomerular molecular-feature clusters in the odorant-receptor class domains of the mouse olfactory bulb. J. Neurophysiol., 103, 3490-3500.

Mattis, J., Tye, K.M., Ferenczi, E.A., Ramakrishnan, C., O'Shea, D.J., Prakash, R., Gunaydin, L.A., Hyun, M., Fenno, L.E., Gradinaru, V., Yizhar, O. \& Deisseroth, K. (2012) Principles for applying optogenetic tools derived from direct comparative analysis of microbial opsins. Nat. Methods, 9, 159-172.

Minichiello, L., Korte, M., Wolfer, D., Kühn, R., Unsicker, K., Cestari, V., Rossi-Arnaud, C., Lipp, H.-P., Bonhoeffer, T. \& Klein, R. (1999) Essential role for TrkB receptors in hippocampus-mediated learning. Neuron, 24, 401-414.

Mizuguchi, R., Naritsuka, H., Mori, K. \& Yoshihara, Y. (2012) Tbr2 deficiency in mitral and tufted cells disrupts excitatory-inhibitory balance of neural circuitry in the mouse olfactory bulb. J. Neurosci., 32, 8831-8844.

Mizutani, K., Yoon, K., Dang, L., Tokunaga, A. \& Gaiano, N. (2007) Differential Notch signalling distinguishes neural stem cells from intermediate progenitors. Nature, 449, 351-355.

Mori, K. \& Yoshihara, Y. (1995) Molecular recognition and olfactory processing in the mammalian olfactory system. Prog. Neurobiol., 45, 585-619.

Mori, K., Takahashi, Y.K., Igarashi, K.M. \& Yamaguchi, M. (2006) Maps of odorant molecular features in the mammalian olfactory bulb. Physiol. Rev., 86, 409-433.

Nagarsheth, M.H., Viehman, A., Lippa, S.M. \& Lippa, C.F. (2006) Notch-1 immunoexpression is increased in Alzheimer's and Pick's disease. J. Neurol. Sci., 244, 111-116.
Nagayama, S., Takahashi, Y.K., Yoshihara, Y. \& Mori, K. (2004) Mitral and tufted cells differ in the decoding manner of odor maps in the rat olfactory bulb. J. Neurophysiol., 91, 2532-2540.

Néant-Fery, M., Pérès, E., Nasrallah, C., Kessner, M., Gribaudo, S., Greer, C., Didier, A., Trembleau, A. \& Caillé, I. (2012) A role for dendritic translation of CaMKII $\alpha$ mRNA in olfactory plasticity. PLoS One, 7, e40133.

Radtke, F., Ferrero, I., Wilson, A., Lees, R., Aguet, M. \& MacDonald, H.R. (2000) Notch1 deficiency dissociates the intrathymic development of dendritic cells and T cells. J. Exp. Med., 191, 1085-1094.

Rahayel, S., Frasnelli, J. \& Joubert, S. (2012) The effect of Alzheimer's disease and Parkinson's disease on olfaction: a meta-analysis. Behav. Brain Res., 231, 60-74.

Sargin, D., Botly, L.C.P., Higgs, G., Marsolais, A., Frankland, P.W., Egan, S.E. \& Josselyn, S.A. (2013) Disrupting Jagged1-Notch signaling impairs spatial memory formation in adult mice. Neurobiol. Learn. Mem., 103, 39-49.

Sato, C., Turkoz, M., Dearborn, J.T., Wozniak, D.F., Kopan, R. \& Hass, M.R. (2012) Loss of RBPj in postnatal excitatory neurons does not cause neurodegeneration or memory impairments in aged mice. PLoS One, 7, e48180.

Schoppa, N.E. (2006) Synchronization of olfactory bulb mitral cells by precisely timed inhibitory inputs. Neuron, 49, 271-283.

Schoppa, N.E. \& Westbrook, G.L. (2001) Glomerulus-specific synchronization of mitral cells in the olfactory bulb. Neuron, 31, 639-651.

Stump, G., Durrer, A., Klein, A.L., Lutolf, S., Suter, U. \& Taylor, V. (2002) Notch1 and its ligands Delta-like and Jagged are expressed and active in distinct cell populations in the postnatal mouse brain. Mech. Develop., 114, 153-159.

Tan, J., Savigner, A., Ma, M. \& Luo, M. (2010) Odor information processing by the olfactory bulb analyzed in gene-targeted mice. Neuron, 65 , $912-926$.

Towbin, H., Staehelin, T. \& Gordon, J. (1979) Electrophoretic transfer of proteins from polyacrylamide gels to nitrocellulose sheets: procedure and some applications. Proc. Natl. Acad. Sci. USA, 76, 4350-4354.

Wang, Y., Chan, S.L., Miele, L., Yao, P.J., Mackes, J., Ingram, D.K., Mattson, M.P. \& Furukawa, K. (2004) Involvement of Notch signaling in hippocampal synaptic plasticity. Proc. Natl. Acad. Sci. USA, 101, 9458-9462.

Willhite, D.C., Nguyen, K.T., Masurkar, A.V., Greer, C.A., Shepherd, G.M. \& Chen, W.R. (2006) Viral tracing identifies distributed columnar organization in the olfactory bulb. Proc. Natl. Acad. Sci. USA, 103, $12592-12597$

Xu, F., Liu, N., Kida, I., Rothman, D.L., Hyder, F. \& Shepherd, G.M. (2003) Odor maps of aldehydes and esters revealed by functional MRI in the glomerular layer of the mouse olfactory bulb. Proc. Natl. Acad. Sci. USA, 100, 11029-11034

Xu, Z., Wang, L., Chen, G., Rao, X. \& Xu, F. (2013) Roles of GSK3 $\beta$ in odor habituation and spontaneous neural activity of the mouse olfactory bulb. PLoS One, 8, e63598.

Zhan, X. \& Heinbockel, T. (2013) The basal forebrain modulates spontaneous activity of principal cells in the main olfactory bulb of anesthetized mice. Front. Neural Circuits, 7, 148.

Zhang, J., Little, C.J., Tremmel, D.M., Yin, J.C.P. \& Wesley, C.S. (2013) Notch-inducible hyperphosphorylated CREB and its ultradian oscillation in long-term memory formation. J. Neurosci., 33, 12825-12834.

Zheng, J., Watanabe, H., Wines-Samuelson, M., Zhao, H., Gridley, T., Kopan, R. \& Shen, J. (2012) Conditional deletion of Notch1 and Notch2 genes in excitatory neurons of postnatal forebrain does not cause neurodegeneration or reduction of Notch mRNAs and proteins. J. Biol. Chem. 287, 20356-20368

Zou, D.-J., Greer, C.A. \& Firestein, S. (2002) Expression pattern of aCaMKII in the mouse main olfactory bulb. J. Comp. Neurol., 443, 226-236. 